Article

\title{
From Tools in Symplectic and Poisson Geometry to J.-M. Souriau's Theories of Statistical Mechanics and Thermodynamics ${ }^{\dagger}$
}

\author{
Charles-Michel Marle
}

Institut de Mathématiques de Jussieu, Université Pierre et Marie Curie, 4, Place Jussieu, 75252 Paris Cedex 05, France; charles-michel.marle@math.cnrs.fr

$\dagger$ In memory of Jean-Marie Souriau (1922-2012).

Academic Editors: Frédéric Barbaresco and Frank Nielsen

Received: 28 July 2016; Accepted: 5 October 2016; Published: 19 October 2016

\begin{abstract}
I present in this paper some tools in symplectic and Poisson geometry in view of their applications in geometric mechanics and mathematical physics. After a short discussion of the Lagrangian an Hamiltonian formalisms, including the use of symmetry groups, and a presentation of the Tulczyjew's isomorphisms (which explain some aspects of the relations between these formalisms), I explain the concept of manifold of motions of a mechanical system and its use, due to J.-M. Souriau, in statistical mechanics and thermodynamics. The generalization of the notion of thermodynamic equilibrium in which the one-dimensional group of time translations is replaced by a multi-dimensional, maybe non-commutative Lie group, is fully discussed and examples of applications in physics are given.
\end{abstract}

Keywords: Lagrangian formalism; Hamiltonian formalism; symplectic manifolds; Poisson structures; symmetry groups; momentum maps; thermodynamic equilibria; generalized Gibbs states

\section{Introduction}

\subsection{Contents of the Paper, Sources and Further Reading}

This paper presents tools in symplectic and Poisson geometry in view of their application in geometric mechanics and mathematical physics. The Lagrangian formalism and symmetries of Lagrangian systems are discussed in Sections 2 and 3, the Hamiltonian formalism and symmetries of Hamiltonian systems in Sections 4 and 5. Section 6 introduces the concepts of Gibbs state and of thermodynamic equilibrium of a mechanical system, and presents several examples. For a monoatomic classical ideal gas, eventually in a gravity field, or a monoatomic relativistic gas the Maxwell-Boltzmann and Maxwell-Jüttner probability distributions are derived. The Dulong and Petit law which governs the specific heat of solids is obtained. Finally Section 7 presents the generalization of the concept of Gibbs state, due to Jean-Marie Souriau, in which the group of time translations is replaced by a (multi-dimensional and eventually non-Abelian) Lie group.

Several books [1-11] discuss, much more fully than in the present paper, the contents of Sections 2-5. The interested reader is referred to these books for detailed proofs of results whose proofs are only briefly sketched here. The recent paper [12] contains detailed proofs of most results presented here in Sections 4 and 5.

The main sources used for Sections 6 and 7 are the book and papers by Jean-Marie Souriau [13-17] and the beautiful small book by Mackey [18].

The Euler-Poincaré equation, which is presented with Lagrangian symmetries at the end of Section 3, is not really related to symmetries of a Lagrangian system, since the Lie algebra which acts 
on the configuration space of the system is not a Lie algebra of symmetries of the Lagrangian. Moreover in its intrinsic form that equation uses the concept of Hamiltonian momentum map presented later, in Section 5. Since the Euler-Poincaré equation is not used in the following sections, the reader can skip the corresponding subsection at his or her first reading.

\subsection{Notations}

The notations used are more or less those generally used now in differential geometry. The tangent and cotangent bundles to a smooth manifold $M$ are denoted by $T M$ and $T^{*} M$, respectively, and their canonical projections by $\tau_{M}: T M \rightarrow M$ and $\pi_{M}: T^{*} M \rightarrow M$. The vector spaces of $k$-multivectors and $k$-forms on $M$ are denoted by $A^{k}(M)$ and $\Omega^{k}(M)$, respectively, with $k \in \mathbb{Z}$ and, of course, $A^{k}(M)=\{0\}$ and $\Omega^{k}(M)=\{0\}$ if $k<0$ and if $k>\operatorname{dim} M, k$-multivectors and $k$-forms being skew-symmetric. The exterior algebras of multivectors and forms of all degrees are denoted by $A(M)=\oplus_{k} A^{k}(M)$ and $\Omega(M)=\oplus_{k} \Omega^{k}(M)$, respectively. The exterior differentiation operator of differential forms on a smooth manifold $M$ is denoted by $\mathrm{d}: \Omega(M) \rightarrow \Omega(M)$. The interior product of a differential form $\eta \in \Omega(M)$ by a vector field $X \in A^{1}(M)$ is denoted by $\mathrm{i}(X) \eta$.

Let $f: M \rightarrow N$ be a smooth map defined on a smooth manifold $M$, with values in another smooth manifold $N$. The pull-back of a form $\eta \in \Omega(N)$ by a smooth map $f: M \rightarrow N$ is denoted by $f^{*} \eta \in \Omega(M)$.

A smooth, time-dependent vector field on the smooth manifold $M$ is a smooth map $X: \mathbb{R} \times M \rightarrow T M$ such that, for each $t \in \mathbb{R}$ and $x \in M, X(t, x) \in T_{x} M$, the vector space tangent to $M$ at $x$. When, for any $x \in M, X(t, x)$ does not depend on $t \in \mathbb{R}, X$ is a smooth vector field in the usual sense, i.e., an element in $A^{1}(M)$. Of course a time-dependent vector field can be defined on an open subset of $\mathbb{R} \times M$ instead than on the whole $\mathbb{R} \times M$. It defines a differential equation

$$
\frac{\mathrm{d} \varphi(t)}{\mathrm{d} t}=X(t, \varphi(t))
$$

said to be associated to $X$. The (full) flow of $X$ is the map $\Psi X$, defined on an open subset of $\mathbb{R} \times \mathbb{R} \times M$, taking its values in $M$, such that for each $t_{0} \in \mathbb{R}$ and $x_{0} \in M$ the parametrized curve $t \mapsto \Psi^{X}\left(t, t_{0}, x_{0}\right)$ is the maximal integral curve of Equation (1) satisfying $\Psi\left(t_{0}, t_{0}, x_{0}\right)=x_{0}$. When $t_{0}$ and $t \in \mathbb{R}$ are fixed, the map $x_{0} \mapsto \Psi^{X}\left(t, t_{0}, x_{0}\right)$ is a diffeomorphism, defined on an open subset of $M$ (which may be empty) and taking its values in another open subset of $M$, denoted by $\Psi_{\left(t, t_{0}\right.}^{X}$. When $X$ is in fact a vector field in the usual sense (not dependent on time), $\Psi_{\left(t, t_{0}\right)}^{X}$ only depends on $t-t_{0}$. Instead of the full flow of $X$ we can use its reduced flow $\Phi^{X}$, defined on an open subset of $\mathbb{R} \times M$ and taking its values in $M$, related to the full flow $\Psi^{X}$ by

$$
\Phi^{X}\left(t, x_{0}\right)=\Psi^{X}\left(t, 0, x_{0}\right), \quad \Psi^{X}\left(t, t_{0}, x_{0}\right)=\Phi^{X}\left(t-t_{0}, x_{0}\right) .
$$

For each $t \in \mathbb{R}$, the map $x_{0} \mapsto \Phi^{X}\left(t, x_{0}\right)=\Psi^{X}\left(t, 0, x_{0}\right)$ is a diffeomorphism, denoted by $\Phi_{t}^{X}$, defined on an open subset of $M$ (which may be empty) onto another open subset of $M$.

When $f: M \rightarrow N$ is a smooth map defined on a smooth manifold $M$, with values in another smooth manifold $N$, there exists a smooth map $T f: T M \rightarrow T N$ called the prolongation of $f$ to vectors, which for each fixed $x \in M$ linearly maps $T_{x} M$ into $T_{f(x)} N$. When $f$ is a diffeomorphism of $M$ onto $N$, $T f$ is an isomorphism of $T M$ onto $T N$. That property allows us to define the canonical lifts of a vector field $X$ in $A^{1}(M)$ to the tangent bundle $T M$ and to the cotangent bundle $T^{*} M$. Indeed, for each $t \in \mathbb{R}$, $\Phi_{t}^{X}$ is a diffeomorphism of an open subset of $M$ onto another open subset of $M$. Therefore $T \Phi_{t}^{X}$ is a diffeomorphism of an open subset of TM onto another open subset of TM. It turns out that when $t$ takes all possible values in $\mathbb{R}$ the set of all diffeomorphisms $T \Phi_{t}^{X}$ is the reduced flow of a vector field $\bar{X}$ on TM, which is the canonical lift of $X$ to the tangent bundle TM.

Similarly, the transpose $\left(T \Phi_{-t}^{X}\right)^{T}$ of $T \Phi_{-t}^{X}$ is a diffeomorphism of an open subset of the cotangent bundle $T^{*} M$ onto another open subset of $T^{*} M$, and when $t$ takes all possible values in $\mathbb{R}$ the set of all 
diffeomorphisms $\left(T \Phi_{-t}^{X}\right)^{T}$ is the reduced flow of a vector field $\widehat{X}$ on $T^{*} M$, which is the canonical lift of $X$ to the cotangent bundle $T^{*} M$.

The canonical lifts of a vector field to the tangent and cotangent bundles are used in Sections 3 and 5. They can be defined too for time-dependent vector fields.

\section{The Lagrangian Formalism}

\subsection{The Configuration Space and the Space of Kinematic States}

The principles of mechanics were stated by the great English mathematician Isaac Newton (1642-1727) in his book Philosophia Naturalis Principia Mathematica published in 1687 [19]. On this basis, a little more than a century later, Joseph Louis Lagrange (1736-1813) in his book Mécanique analytique [20] derived the equations (today known as the Euler-Lagrange equations) which govern the motion of a mechanical system made of any number of material points or rigid material bodies interacting between them by very general forces, and eventually submitted to external forces.

In modern mathematical language, these equations are written on the configuration space and on the space of kinematic states of the considered mechanical system. The configuration space is a smooth $n$-dimensional manifold $N$ whose elements are all the possible configurations of the system (a configuration being the position in space of all parts of the system). The space of kinematic states is the tangent bundle $T N$ to the configuration space, which is $2 n$-dimensional. Each element of the space of kinematic states is a vector tangent to the configuration space at one of its elements, i.e., at a configuration of the mechanical system, which describes the velocity at which this configuration changes with time. In local coordinates a configuration of the system is determined by the $n$ coordinates $x^{1}, \ldots, x^{n}$ of a point in $N$, and a kinematic state by the $2 n$ coordinates $x^{1}, \ldots, x^{n}, v^{1}, \ldots v^{n}$ of a vector tangent to $N$ at some element in $N$.

\subsection{The Euler-Lagrange Equations}

When the mechanical system is conservative, the Euler-Lagrange equations involve a single real valued function $L$ called the Lagrangian of the system, defined on the product of the real line $\mathbb{R}$ (spanned by the variable $t$ representing the time) with the manifold $T N$ of kinematic states of the system. In local coordinates, the Lagrangian $L$ is expressed as a function of the $2 n+1$ variables, $t, x^{1}, \ldots, x^{n}, v^{1}, \ldots, v^{n}$ and the Euler-Lagrange equations have the remarkably simple form

$$
\frac{\mathrm{d}}{\mathrm{d} t}\left(\frac{\partial L}{\partial v^{i}}(t, x(t), v(t))\right)-\frac{\partial L}{\partial x^{i}}(t, x(t), v(t))=0, \quad 1 \leq i \leq n,
$$

where $x(t)$ stands for $x^{1}(t), \ldots, x^{n}(t)$ and $v(t)$ for $v^{1}(t), \ldots, v^{n}(t)$ with, of course,

$$
v^{i}(t)=\frac{\mathrm{d} x^{i}(t)}{\mathrm{d} t}, \quad 1 \leq i \leq n
$$

\subsection{Hamilton's Principle of Stationary Action}

The great Irish mathematician William Rowan Hamilton (1805-1865) observed [21,22] that the Euler-Lagrange equations can be obtained by applying the standard techniques of Calculus of Variations, due to Leonhard Euler (1707-1783) and Joseph Louis Lagrange, to the action integral (Lagrange observed that fact before Hamilton, but in the last edition of his book he chose to derive the Euler-Lagrange equations by application of the principle of virtual works, using a very clever evaluation of the virtual work of inertial forces for a smooth infinitesimal variation of the motion).

$$
I_{L}(\gamma)=\int_{t_{0}}^{t_{1}} L(t, x(t), v(t)) \mathrm{d} t, \quad \text { with } v(t)=\frac{\mathrm{d} x(t)}{\mathrm{d} t}
$$


where $\gamma:\left[t_{0}, t_{1}\right] \rightarrow N$ is a smooth curve in $N$ parametrized by the time $t$. These equations express the fact that the action integral $I_{L}(\gamma)$ is stationary with respect to any smooth infinitesimal variation of $\gamma$ with fixed end-points $\left(t_{0}, \gamma\left(t_{0}\right)\right)$ and $\left(t_{1}, \gamma\left(t_{1}\right)\right)$. This fact is today called Hamilton's principle of stationary action. The reader interested in Calculus of Variations and its applications in mechanics and physics is referred to the books [23-25].

\subsection{The Euler-Cartan Theorem}

The Lagrangian formalism is the use of Hamilton's principle of stationary action for the derivation of the equations of motion of a system. It is widely used in mathematical physics, often with more general Lagrangians involving more than one independent variable and higher order partial derivatives of dependent variables. For simplicity I will consider here only the Lagrangians of (maybe time-dependent) conservative mechanical systems.

An intrinsic geometric expression of the Euler-Lagrange equations, wich does not use local coordinates, was obtained by the great French mathematician Élie Cartan (1869-1951). Let us introduce the concepts used by the statement of this theorem.

Definition 1. Let $N$ be the configuration space of a mechanical system and let its tangent bundle TN be the space of kinematic states of that system. We assume that the evolution with time of the state of the system is governed by the Euler-Lagrange equations for a smooth, maybe time-dependent Lagrangian $L: \mathbb{R} \times T N \rightarrow \mathbb{R}$.

1. The cotangent bundle $T^{*} N$ is called the phase space of the system.

2. The map $\mathcal{L}_{L}: \mathbb{R} \times T N \rightarrow T^{*} N$

$$
\mathcal{L}_{L}(t, v)=\mathrm{d}_{\mathrm{vert}} L(t, v), \quad t \in \mathbb{R}, v \in T N,
$$

where $\mathrm{d}_{\mathrm{vert}} L(t, v)$ is the vertical differential of $L$ at $(t, v)$, i.e., the differential at $v$ of the the map $w \mapsto L(t, w)$, with $w \in \tau_{N}^{-1}\left(\tau_{N}(v)\right)$, is called the Legendre map associated to $L$.

3. The map $E_{L}: \mathbb{R} \times T N \rightarrow \mathbb{R}$ given by

$$
E_{L}(t, v)=\left\langle\mathcal{L}_{L}(t, v), v\right\rangle-L(t, v), \quad t \in \mathbb{R}, v \in T N,
$$

is called the the energy function associated to $L$.

4. The 1-form on $\mathbb{R} \times T N$

$$
\widehat{\omega}_{L}=\mathcal{L}_{L}^{*} \theta_{N}-E_{L}(t, v) \mathrm{d} t,
$$

where $\theta_{N}$ is the Liouville 1-form on $T^{*} N$, is called the Euler-Poincare 1-form.

Theorem 1 (Euler-Cartan Theorem). A smooth curve $\gamma:\left[t_{0}, t_{1}\right] \rightarrow N$ parametrized by the time $t \in\left[t_{0}, t_{1}\right]$ is a solution of the Euler-Lagrange equations if and only if, for each $t \in\left[t_{0}, t_{1}\right]$ the derivative with respect to $t$ of the map $t \mapsto\left(t, \frac{\mathrm{d} \gamma(t)}{\mathrm{d} t}\right)$ belongs to the kernel of the 2 -form $\mathrm{d} \widehat{\omega}_{L}$, in other words if and only if

$$
\mathrm{i}\left(\frac{\mathrm{d}}{\mathrm{d} t}\left(t, \frac{\mathrm{d} \gamma(t)}{\mathrm{d} t}\right)\right) \mathrm{d} \widehat{\omega}_{L}\left(t, \frac{\mathrm{d} \gamma(t)}{\mathrm{d} t}\right)=0 .
$$

The interested reader will find the proof of that theorem in [26], (Theorem 2.2, Chapter IV, p. 262) or, for hyper-regular Lagrangians (an additional assumption which in fact, is not necessary) in [27], Chapter IV, Theorem 2.1, p. 167.

Remark 1. In his book [14], Jean-Marie Souriau uses a slightly different terminology: for him the odd-dimensional space $\mathbb{R} \times T N$ is the evolution space of the system, and the exact 2-form $\mathrm{d} \widehat{\omega}_{L}$ on that space is the Lagrange form. He defines that 2-form in a setting more general than that of the Lagrangian formalism. 


\section{Lagrangian Symmetries}

\subsection{Assumptions and Notations}

In this section $N$ is the configuration space of a conservative Lagrangian mechanical system with a smooth, maybe time dependent Lagrangian $L: \mathbb{R} \times T N \rightarrow \mathbb{R}$. Let $\widehat{\omega}_{L}$ be the Poincaré-Cartan 1-form on the evolution space $\mathbb{R} \times T N$.

Several kinds of symmetries can be defined for such a system. Very often, they are special cases of infinitesimal symmetries of the Poincaré-Cartan form, which play an important part in the famous Noether theorem.

Definition 2. An infinitesimal symmetry of the Poincaré-Cartan form $\widehat{\omega}_{L}$ is a vector field $Z$ on $\mathbb{R} \times T N$ such that

$$
\mathcal{L}(Z) \widehat{\omega}_{L}=0,
$$

$\mathcal{L}(Z)$ denoting the Lie derivative of differential forms with respect to $Z$.

\section{Example 1.}

1. Let us assume that the Lagrangian $L$ does not depend on the time $t \in \mathbb{R}$, i.e., is a smooth function on $T N$. The vector field on $\mathbb{R} \times T N$ denoted by $\frac{\partial}{\partial t}$, whose projection on $\mathbb{R}$ is equal to 1 and whose projection on $T N$ is 0 , is an infinitesimal symmetry of $\widehat{\omega}_{L}$.

2. Let $X$ be a smooth vector field on $N$ and $\bar{X}$ be its canonical lift to the tangent bundle $T N$. We still assume that $L$ does not depend on the time $t$. Moreover we assume that $\bar{X}$ is an infinitesimal symmetry of the Lagrangian $L$, i.e., that $\mathcal{L}(\bar{X}) L=0$. Considered as a vector field on $\mathbb{R} \times T N$ whose projection on the factor $\mathbb{R}$ is $0, \bar{X}$ is an infinitesimal symmetry of $\widehat{\omega}_{L}$.

\subsection{The Noether Theorem in Lagrangian Formalism}

Theorem 2 (E. Noether's Theorem in Lagrangian Formalism). Let $\mathrm{Z}$ be an infinitesimal symmetry of the Poincaré-Cartan form $\widehat{\omega}_{L}$. For each possible motion $\gamma:\left[t_{0}, t_{1}\right] \rightarrow N$ of the Lagrangian system, the function $\mathrm{i}(Z) \widehat{\omega}_{L}$, defined on $\mathbb{R} \times T N$, keeps a constant value along the parametrized curve $t \mapsto\left(t, \frac{\mathrm{d} \gamma(t)}{\mathrm{d} t}\right)$.

Proof. Let $\gamma:\left[t_{0}, t_{1}\right] \rightarrow N$ be a motion of the Lagrangian system, i.e., a solution of the Euler-Lagrange equations. The Euler-Cartan Theorem 1 proves that, for any $t \in\left[t_{0}, t_{1}\right]$,

$$
\mathrm{i}\left(\frac{\mathrm{d}}{\mathrm{d} t}\left(t, \frac{\mathrm{d} \gamma(t)}{\mathrm{d} t}\right)\right) \mathrm{d} \widehat{\omega}_{L}\left(t, \frac{\mathrm{d} \gamma(t)}{\mathrm{d} t}\right)=0 .
$$

Since $Z$ is an infinitesimal symmetry of $\widehat{\omega}_{L}$,

$$
\mathcal{L}(Z) \widehat{\omega}_{L}=0 .
$$

Using the well known formula relating the Lie derivative, the interior product and the exterior derivative

$$
\mathcal{L}(Z)=\mathrm{i}(Z) \circ \mathrm{d}+\mathrm{d} \circ \mathrm{i}(Z)
$$


we can write

$$
\begin{aligned}
\frac{\mathrm{d}}{\mathrm{d} t}\left(\mathrm{i}(Z) \widetilde{\omega}_{L}\left(t, \frac{\mathrm{d} \gamma(t)}{\mathrm{d} t}\right)\right) & =\left\langle\operatorname{di}(Z) \widehat{\omega}_{L}, \frac{\mathrm{d}}{\mathrm{d} t}\left(t, \frac{\mathrm{d} \gamma(t)}{\mathrm{d} t}\right)\right\rangle \\
& =-\left\langle\mathrm{i}(Z) \mathrm{d} \widehat{\omega}_{L}, \frac{\mathrm{d}}{\mathrm{d} t}\left(t, \frac{\mathrm{d} \gamma(t)}{\mathrm{d} t}\right)\right\rangle \\
& =0 .
\end{aligned}
$$

Example 2. When the Lagrangian $L$ does not depend on time, application of Emmy Noether's theorem to the vector field $\frac{\partial}{\partial t}$ shows that the energy $E_{L}$ remains constant during any possible motion of the system, since $\mathrm{i}\left(\frac{\partial}{\partial t}\right) \widehat{\omega}_{L}=-E_{L}$.

\section{Remark 2.}

1. Theorem 2 is due to the German mathematician Emmy Noether (1882-1935), who proved it under much more general assumptions than those used here. For a very nice presentation of Emmy Noether's theorems in a much more general setting and their applications in mathematical physics, interested readers are referred to the very nice book by Yvette Kosmann-Schwarzbach [28].

2. Several generalizations of the Noether theorem exist. For example, if instead of being an infinitesimal symmetry of $\widehat{\omega}_{L}$, i.e., instead of satisfying $\mathcal{L}(Z) \widehat{\omega}_{L}=0$ the vector field $Z$ satisfies

$$
\mathcal{L}(Z) \widehat{\omega}_{L}=\mathrm{d} f,
$$

where $f: \mathbb{R} \times T M \rightarrow \mathbb{R}$ is a smooth function, which implies of course $\mathcal{L}(Z)\left(\mathrm{d} \widehat{\omega}_{L}\right)=0$, the function

$$
\mathrm{i}(Z) \widehat{\omega}_{L}-f
$$

keeps a constant value along $t \mapsto\left(t, \frac{\mathrm{d} \gamma(t)}{\mathrm{d} t}\right)$.

\subsection{The Lagrangian Momentum Map}

The Lie bracket of two infinitesimal symmetries of $\widehat{\omega}_{L}$ is too an infinitesimal symmetry of $\widehat{\omega}_{L}$. Let us therefore assume that there exists a finite-dimensional Lie algebra of vector fields on $\mathbb{R} \times T N$ whose elements are infinitesimal symmetries of $\widehat{\omega}_{L}$.

Definition 3. Let $\psi: \mathcal{G} \rightarrow A^{1}(\mathbb{R} \times T N)$ be a Lie algebras homomorphism of a finite-dimensional real Lie algebra $\mathcal{G}$ into the Lie algebra of smooth vector fields on $\mathbb{R} \times T N$ such that, for each $X \in \mathcal{G}, \psi(X)$ is an infinitesimal symmetry of $\widehat{\omega}_{L}$. The Lie algebras homomorphism $\psi$ is said to be a Lie algebra action on $\mathbb{R} \times T N$ by infinitesimal symmetries of $\widehat{\omega}_{L}$. The map $K_{L}: \mathbb{R} \times T N \rightarrow \mathcal{G}^{*}$, which takes its values in the dual $\mathcal{G}^{*}$ of the Lie algebra $\mathcal{G}$, defined by

$$
\left\langle K_{L}(t, v), X\right\rangle=\mathrm{i}(\psi(X)) \widehat{\omega}_{L}(t, v), \quad X \in \mathcal{G}, \quad(t, v) \in \mathbb{R} \times T N,
$$

is called the Lagrangian momentum of the Lie algebra action $\psi$.

Corollary 1 (of E. Noether's Theorem). Let $\psi: \mathcal{G} \rightarrow A^{1}(\mathbb{R} \times T M)$ be an action of a finite-dimensional real Lie algebra $\mathcal{G}$ on the evolution space $\mathbb{R} \times T N$ of a conservative Lagrangian system, by infinitesimal symmetries of the Poincaré-Cartan form $\widehat{\omega}_{L}$. For each possible motion $\gamma:\left[t_{0}, t_{1}\right] \rightarrow N$ of that system, the Lagrangian momentum map $K_{L}$ keeps a constant value along the parametrized curve $t \mapsto\left(t, \frac{\mathrm{d} \gamma(t)}{\mathrm{d} t}\right)$. 
Proof. Since for each $X \in \mathcal{G}$ the function $(t, v) \mapsto\left\langle K_{L}(t, v), X\right\rangle$ keeps a constant value along the parametrized curve $t \mapsto\left(t, \frac{\mathrm{d} \gamma(t)}{\mathrm{d} t}\right)$, the map $K_{L}$ itself keeps a constant value along that parametrized curve.

Example 3. Let us assume that the Lagrangian $L$ does not depend explicitly on the time $t$ and is invariant by the canonical lift to the tangent bundle of the action on $N$ of the six-dimensional group of Euclidean diplacements (rotations and translations) of the physical space. The corresponding infinitesimal action of the Lie algebra of infinitesimal Euclidean displacements (considered as an action on $\mathbb{R} \times T N$, the action on the factor $\mathbb{R}$ being trivial) is an action by infinitesimal symmetries of $\widehat{\omega}_{L}$. The six components of the Lagrangian momentum map are the three components of the total linear momentum and the three components of the total angular momentum.

Remark 3. These results are valid without any assumption of hyper-regularity of the Lagrangian.

\subsection{The Euler-Poincaré Equation}

In a short Note [29] published in 1901, the great french mathematician Henri Poincaré (1854-1912) proposed a new formulation of the equations of mechanics.

Let $N$ be the configuration manifold of a conservative Lagrangian system, with a smooth Lagrangian $L: T N \rightarrow \mathbb{R}$ which does not depend explicitly on time. Poincaré assumes that there exists an homomorphism $\psi$ of a finite-dimensional real Lie algebra $\mathcal{G}$ into the Lie algebra $A^{1}(N)$ of smooth vector fields on $N$, such that for each $x \in N$, the values at $x$ of the vector fields $\psi(X)$, when $X$ varies in $\mathcal{G}$, completely fill the tangent space $T_{x} N$. The action $\psi$ is then said to be locally transitive.

Of course these assumptions imply $\operatorname{dim} \mathcal{G} \geq \operatorname{dim} N$.

Under these assumptions, Henri Poincare proved that the equations of motion of the Lagrangian system could be written on $N \times \mathcal{G}$ or on $N \times \mathcal{G}^{*}$, where $\mathcal{G}^{*}$ is the dual of the Lie algebra $\mathcal{G}$, instead of on the tangent bundle $T N$. When $\operatorname{dim} \mathcal{G}=\operatorname{dim} N$ (which can occur only when the tangent bundle $T N$ is trivial) the obtained equation, called the Euler-Poincaré equation, is perfectly equivalent to the Euler-Lagrange equations and may, in certain cases, be easier to use. But when $\operatorname{dim} \mathcal{G}>\operatorname{dim} N$, the system made by the Euler-Poincare equation is underdetermined.

Let $\gamma:\left[t_{0}, t_{1}\right] \rightarrow N$ be a smooth parametrized curve in $N$. Poincaré proves that there exists a smooth curve $V:\left[t_{0}, t_{1}\right] \rightarrow \mathcal{G}$ in the Lie algebra $\mathcal{G}$ such that, for each $t \in\left[t_{0}, t_{1}\right]$,

$$
\psi(V(t))(\gamma(t))=\frac{\mathrm{d} \gamma(t)}{\mathrm{d} t} .
$$

When $\operatorname{dim} \mathcal{G}>\operatorname{dim} N$ the smooth curve $V$ in $\mathcal{G}$ is not uniquely determined by the smooth curve $\gamma$ in $N$. However, instead of writing the second-order Euler-Lagrange differential equations on $T N$ satisfied by $\gamma$ when this curve is a possible motion of the Lagrangian system, Poincaré derives a first order differential equation for the curve $V$ and proves that it is satisfied, together with Equation (2), if and only if $\gamma$ is a possible motion of the Lagrangian system.

Let $\varphi: N \times \mathcal{G} \rightarrow T N$ and $\bar{L}: N \times \mathcal{G} \rightarrow \mathbb{R}$ be the maps

$$
\varphi(x, X)=\psi(X)(x), \quad \bar{L}(x, X)=L \circ \varphi(x, X) .
$$

We denote by $\mathrm{d}_{1} \bar{L}: N \times \mathcal{G} \rightarrow T^{*} N$ and by $d_{2} \bar{L}: N \times \mathcal{G} \rightarrow \mathcal{G}^{*}$ the partial differentials of $\bar{L}: N \times \mathcal{G} \rightarrow \mathbb{R}$ with respect to its first variable $x \in N$ and with respect to its second variable $X \in \mathcal{G}$.

The map $\varphi: N \times \mathcal{G} \rightarrow T N$ is a surjective vector bundles morphism of the trivial vector bundle $N \times \mathcal{G}$ into the tangent bundle $T N$. Its transpose $\varphi^{T}: T^{*} N \rightarrow N \times \mathcal{G}^{*}$ is therefore an injective vector bundles morphism, which can be written

$$
\varphi^{T}(\xi)=\left(\pi_{N}(\xi), J(\xi)\right),
$$


where $\pi_{N}: T^{*} N \rightarrow N$ is the canonical projection of the cotangent bundle and $J: T^{*} N \rightarrow \mathcal{G}^{*}$ is a smooth map whose restriction to each fibre $T_{x}^{*} N$ of the cotangent bundle is linear, and is the transpose of the map $X \mapsto \varphi(x, X)=\psi(X)(x)$.

Remark 4. The homomorphism $\psi$ of the Lie algebra $\mathcal{G}$ into the Lie algebra $A^{1}(N)$ of smooth vector fields on $N$ is an action of that Lie algebra, in the sense defined below Definition 11. That action can be canonically lifted into a Hamiltonian action of $\mathcal{G}$ on $T^{*} N$, endowed with its canonical symplectic form $\mathrm{d} \theta_{N}$ Definition 13 . The map $J$ is in fact a Hamiltonian momentum map for that Hamiltonian action Proposition 5.

Let $\mathcal{L}_{L}=\mathrm{d}_{\text {vert }} L: T N \rightarrow T^{*} N$ be the Legendre map defined in Definition 1 .

Theorem 3 (Euler-Poincaré Equation). With the above defined notations, let $\gamma:\left[t_{0}, t_{1}\right] \rightarrow N$ be a smooth parametrized curve in $N$ and $V:\left[t_{0}, t_{1}\right] \rightarrow \mathcal{G}$ be a smooth parametrized curve such that, for each $t \in\left[t_{0}, t_{1}\right]$,

$$
\psi(V(t))(\gamma(t))=\frac{\mathrm{d} \gamma(t)}{\mathrm{d} t} .
$$

The curve $\gamma$ is a possible motion of the Lagrangian system if and only if $V$ satisfies the equation

$$
\left(\frac{\mathrm{d}}{\mathrm{d} t}-\operatorname{ad}_{V(t)}^{*}\right)\left(J \circ \mathcal{L}_{L} \circ \varphi(\gamma(t), V(t))\right)-J \circ \mathrm{d}_{1} \bar{L}(\gamma(t), V(t))=0 .
$$

The interested reader will find a proof of that theorem in local coordinates in the original Note by Poincaré [29]. More intrinsic proofs can be found in [12,30]. Another proof is possible, in which that theorem is deduced from the Euler-Cartan Theorem 1.

Remark 5. Equation (3) is called the compatibility condition and Equation (4) is the Euler-Poincare equation. It can be written under the equivalent form

$$
\left(\frac{\mathrm{d}}{\mathrm{d} t}-\operatorname{ad}_{V(t)}^{*}\right)\left(\mathrm{d}_{2} \bar{L}(\gamma(t), V(t))\right)-J \circ \mathrm{d}_{1} \bar{L}(\gamma(t), V(t))=0 .
$$

Examples of applications of the Euler-Poincaré equation can be found in $[5,6,12,30]$ and, for an application in thermodynamics, [31].

\section{The Hamiltonian Formalism}

The Lagrangian formalism can be applied to any smooth Lagrangian. Its application yields second order differential equations on $\mathbb{R} \times N$ (in local coordinates, the Euler-Lagrange equations) which in general are not solved with respect to the second order derivatives of the unknown functions with respect to time. The classical existence and unicity theorems for the solutions of differential equations (such as the Cauchy-Lipschitz theorem) therefore cannot be applied to these equations.

Under the additional assumption that the Lagrangian is hyper-regular, a very clever change of variables discovered by William Rowan Hamilton (Lagrange obtained however Hamilton's equations before Hamilton, but only in a special case, for the slow "variations of constants" such as the orbital parameters of planets in the solar system [32,33]). Hamilton [21,22] allows a new formulation of these equations in the framework of symplectic geometry. The Hamiltonian formalism discussed below is the use of these new equations. It was later generalized independently of the Lagrangian formalism. 


\subsection{Hyper-Regular Lagrangians}

Assumptions Made in this Section

We consider in this section a smooth, maybe time-dependent Lagrangian $L: \mathbb{R} \times T N \rightarrow \mathbb{R}$, which is such that the Legendre map Definition $1 \mathcal{L}_{L}: \mathbb{R} \times T N \rightarrow T^{*} N$ satisfies the following property: for each fixed value of the time $t \in \mathbb{R}$, the map $v \mapsto \mathcal{L}_{L}(t, v)$ is a smooth diffeomorphism of the tangent bundle $T N$ onto the cotangent bundle $T^{*} N$. An equivalent assumption is the following: the $\operatorname{map}\left(\operatorname{id}_{\mathbb{R}}, \mathcal{L}_{L}\right):(t, v) \mapsto\left(t, \mathcal{L}_{L}(t, v)\right)$ is a smooth diffeomorphism of $\mathbb{R} \times T N$ onto $\mathbb{R} \times T^{*} N$. The Lagrangian $L$ is then said to be hyper-regular. The equations of motion can be written on $\mathbb{R} \times T^{*} N$ instead of $\mathbb{R} \times T N$.

Definition 4. Under the assumption Section 4.1, the function $H_{L}: \mathbb{R} \times T^{*} N \rightarrow \mathbb{R}$ given by

$$
H_{L}(t, p)=E_{L} \circ\left(\operatorname{id}_{\mathbb{R}}, \mathcal{L}_{L}\right)^{-1}(t, p), \quad t \in \mathbb{R}, p \in T^{*} N,
$$

$\left(E_{L}: \mathbb{R} \times T N \rightarrow \mathbb{R}\right.$ being the energy function defined in Definition 1 ) is called the Hamiltonian associated to the hyper-regular Lagrangian $L$.

The 1-form defined on $\mathbb{R} \times T^{*} N$

$$
\widehat{\omega}_{H_{L}}=\theta_{N}-H_{L} \mathrm{~d} t
$$

where $\theta_{N}$ is the Liouville 1-form on $T^{*} N$, is called the Poincaré-Cartan 1-form in the Hamiltonian formalism.

Remark 6. The Poincaré-Cartan 1-form $\widehat{\omega}_{L}$ on $\mathbb{R} \times T N$, defined in Definition 1, is the pull-back, by the diffeomorphism $\left(\mathrm{id}_{\mathbb{R}}, \mathcal{L}_{L}\right): \mathbb{R} \times T N \rightarrow \mathbb{R} \times T^{*} N$, of the Poincaré-Cartan 1-form $\widehat{\omega}_{H_{L}}$ in the Hamiltonian formalism on $\mathbb{R} \times T^{*} N$ defined above.

\subsection{Presymplectic Manifolds}

Definition 5. A presymplectic form on a smooth manifold $M$ is a 2-form $\omega$ on $M$ which is closed, i.e., such that $\mathrm{d} \omega=0$. A manifold $M$ equipped with a presymplectic form $\omega$ is called a presymplectic manifold and denoted by $(M, \omega)$. The kernel ker $\omega$ of a presymplectic form $\omega$ defined on a smooth manifold $M$ is the set of vectors $v \in T M$ such that $\mathrm{i}(v) \omega=0$.

Remark 7. A symplectic form $\omega$ on a manifold $M$ is a presymplectic form which, moreover, is non-degenerate, i.e., such that for each $x \in M$ and each non-zero vector $v \in T_{x} M$, there exists another vector $w \in T_{x} M$ such that $\omega(x)(v, w) \neq 0$. Or in other words, a presymplectic form $\omega$ whose kernel is the set of null vectors.

The kernel of a presymplectic form $\omega$ on a smooth manifold $M$ is a vector sub-bundle of TM if and only if for each $x \in M$, the vector subspace $T_{x} M$ of vectors $v \in T_{x} M$ which satisfy $\mathrm{i}(v) \omega=0$ is of a fixed dimension, the same for all points $x \in M$. A presymplectic form which satisfies that condition is said to be of constant rank.

Proposition 1. Let $\omega$ be a presymplectic form of constant rank Remark 7 on a smooth manifold $M$. The kernel ker $\omega$ of $\omega$ is a completely integrable vector sub-bundle of $T M$, which defines a foliation $\mathcal{F}_{\omega}$ of $M$ into connected immersed submanifolds which, at each point of $M$, have the fibre of ker $\omega$ at that point as tangent vector space.

We now assume in addition that this foliation is simple, i.e., such that the set of leaves of $\mathcal{F}_{\omega}$, denoted by $M / \operatorname{ker} \omega$, has a smooth manifold structure for which the canonical projection $p: M \rightarrow M / \operatorname{ker} \omega$ (which associates to each point $x \in M$ the leaf which contains $x$ ) is a smooth submersion. There exists on $M / \operatorname{ker} \omega$ a unique symplectic form $\omega_{r}$ such that

$$
\omega=p^{*} \omega_{r}
$$


Proof. Since $\mathrm{d} \omega=0$, the fact that $\operatorname{ker} \omega$ is completely integrable is an immediate consequence of the Frobenius' theorem ([27], Chapter III, Theorem 5.1, p. 132). The existence and unicity of a symplectic form $\omega_{r}$ on $M / \operatorname{ker} \omega$ such that $\omega=p^{*} \omega_{r}$ results from the fact that $M / \operatorname{ker} \omega$ is built by quotienting $M$ by the kernel of $\omega$.

Presymplectic Manifolds in Mechanics

Let us go back to the assumptions and notations of Section 4.1. We have seen in Remark 6 that the Poincaré-Cartan 1-form in Hamiltonian formalism $\widehat{\omega}_{H_{L}}$ on $\mathbb{R} \times T^{*} N$ and the Poincaré-Cartan 1-form in Lagrangian formalism $\widehat{\widehat{\omega}}_{L}$ on $\mathbb{R} \times T N$ are related by

$$
\widehat{\omega}_{L}=\left(\operatorname{id}_{\mathbb{R}}, \mathcal{L}_{L}\right)^{*} \widehat{\omega}_{H_{L}}
$$

Their exterior differentials $\mathrm{d} \widehat{\omega}_{L}$ and $\mathrm{d} \widehat{\omega}_{H_{L}}$ both are presymplectic 2-forms on the odd-dimensional manifolds $\mathbb{R} \times T N$ and $\mathbb{R} \times T^{*} N$, respectively. At any point of these manifolds, the kernels of these closed 2-forms are one-dimensional. They therefore Proposition 1 determine foliations into smooth curves of these manifolds. The Euler-Cartan Theorem 1 shows that each of these curves is a possible motion of the system, described either in the Lagrangian formalism, or in the Hamiltonian formalism, respectively.

The set of all possible motions of the system, called by Jean-Marie Souriau the manifold of motions of the system, is described by the quotient $(\mathbb{R} \times T N) /$ ker $\mathrm{d} \widehat{\omega}_{L}$ in the Lagrangian formalism, and by the quotient $\left(\mathbb{R} \times T^{*} N\right) / \operatorname{ker} \mathrm{d} \widehat{\omega}_{H_{L}}$ in the Hamiltonian formalism. Both are (maybe non-Hausdorff) symplectic manifolds, the projections on these quotient manifolds of the presymplectic forms $\mathrm{d} \widehat{\Phi}_{L}$ and $\mathrm{d} \widehat{\omega}_{H_{L}}$ both being symplectic forms. Of course the diffeomorphism $\left(\operatorname{id}_{\mathbb{R}}, \mathcal{L}_{L}\right): \mathbb{R} \times T N \rightarrow \mathbb{R} \times T^{*} N$ projects onto a symplectomorphism between the Lagrangian and Hamiltonian descriptions of the manifold of motions of the system.

\subsection{The Hamilton Equation}

Proposition 2. Let $N$ be the configuration manifold of a Lagrangian system whose Lagrangian $L: \mathbb{R} \times T N \rightarrow \mathbb{R}$, maybe time-dependent, is smooth and hyper-regular, and $H_{L}: \mathbb{R} \times T^{*} N \rightarrow \mathbb{R}$ be the associated Hamiltonian Definition 4. Let $\varphi:\left[t_{0}, t_{1}\right] \rightarrow N$ be a smooth curve parametrized by the time $t \in\left[t_{0}, t_{1}\right]$, and let $\psi:\left[t_{0}, t_{1}\right] \rightarrow T^{*} N$ be the parametrized curve in $T^{*} N$

$$
\psi(t)=\mathcal{L}_{L}\left(t, \frac{\mathrm{d} \gamma(t)}{\mathrm{d} t}\right), \quad t \in\left[t_{0}, t_{1}\right]
$$

where $\mathcal{L}_{L}: \mathbb{R} \times T N \rightarrow T^{*} N$ is the Legendre map Definition 1 .

The parametrized curve $t \mapsto \gamma(t)$ is a motion of the system if and only if the parametrized curve $t \mapsto \psi(t)$ satisfies the equatin, called the Hamilton equation,

$$
\mathrm{i}\left(\frac{\mathrm{d} \psi(t)}{\mathrm{d} t}\right) \mathrm{d} \theta_{N}=-\mathrm{d} H_{L t}
$$

where $\mathrm{d} H_{L t}=\mathrm{d} H_{L}-\frac{\partial H_{L}}{\partial t} \mathrm{~d} t$ is the differential of the function $H_{L t}: T^{*} N \rightarrow \mathbb{R}$ in which the time $t$ is considered as a parameter with respect to which there is no differentiation.

When the parametrized curve $\psi$ satisfies the Hamilton equation stated above, it satisfies too the equation, called the energy equation

$$
\frac{\mathrm{d}}{\mathrm{d} t}\left(H_{L}(t, \psi(t))\right)=\frac{\partial H_{L}}{\partial t}(t, \psi(t)) .
$$

Proof. These results directly follow from the Euler-Cartan Theorem 1. 
Remark 8. The 2-form $\mathrm{d} \theta_{N}$ is a symplectic form on the cotangent bundle $T^{*} N$, called its canonical symplectic form. We have shown that when the Lagrangian $L$ is hyper-regular, the equations of motion can be written in three equivalent manners:

1. as the Euler-Lagrange equations on $\mathbb{R} \times T M$,

2. as the equations given by the kernels of the presymplectic forms $\mathrm{d} \widehat{\omega}_{L}$ or $\mathrm{d} \widehat{\omega}_{H_{L}}$ which determine the foliations into curves of the evolution spaces $\mathbb{R} \times T M$ in the Lagrangian formalism, or $\mathbb{R} \times T^{*} M$ in the Hamiltonian formalism,

3. as the Hamilton equation associated to the Hamiltonian $H_{L}$ on the symplectic manifold $\left(T^{*} N, \mathrm{~d} \theta_{N}\right)$, often called the phase space of the system.

\subsubsection{The Tulczyjew Isomorphisms}

Around 1974, Tulczyjew [34,35] discovered ( $\beta_{N}$ was probably known long before 1974, but I believe that $\alpha_{N}$, much more hidden, was noticed by Tulczyjew for the first time) two remarkable vector bundles isomorphisms $\alpha_{N}: T T^{*} N \rightarrow T^{*} T N$ and $\beta_{N}: T T^{*} N \rightarrow T^{*} T^{*} N$.

The first one $\alpha_{N}$ is an isomorphism of the bundle $\left(T T^{*} N, T \pi_{N}, T N\right)$ onto the bundle $\left(T^{*} T N, \pi_{T N}, T N\right)$, while the second $\beta_{N}$ is an isomorphism of the bundle $\left(T T^{*} N, \tau_{T^{*} N}, T^{*} N\right)$ onto the bundle $\left(T^{*} T^{*} N, \pi_{T^{*} N}, T^{*} N\right)$. The diagram below is commutative.

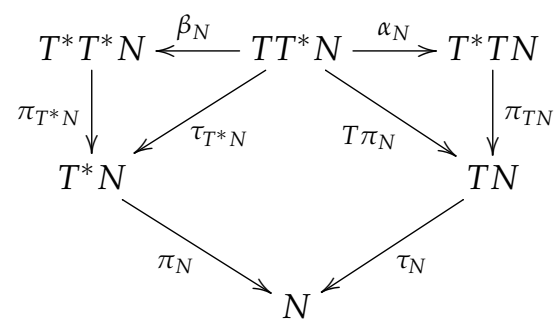

Since they are the total spaces of cotangent bundles, the manifolds $T^{*} T N$ and $T^{*} T^{*} N$ are endowed with the Liouville 1-forms $\theta_{T N}$ and $\theta_{T^{*} N}$, and with the canonical symplectic forms $\mathrm{d} \theta_{T N}$ and $\mathrm{d} \theta_{T^{*} N}$, respectively. Using the isomorphisms $\alpha_{N}$ and $\beta_{N}$, we can therefore define on $T T^{*} N$ two 1-forms $\alpha_{N}^{*} \theta_{T N}$ and $\beta_{N}^{*} \theta_{T^{*} N}$, and two symplectic 2-forms $\alpha_{N}^{*}\left(\mathrm{~d} \theta_{T N}\right)$ and $\beta_{N}^{*}\left(\mathrm{~d} \theta_{T^{*} N}\right)$. The very remarkable property of the isomorphisms $\alpha_{N}$ and $\beta_{N}$ is that the two symplectic forms so obtained on $\mathrm{TT}^{*} N$ are equal:

$$
\alpha_{N}^{*}\left(\mathrm{~d} \theta_{T N}\right)=\beta_{N}^{*}\left(\mathrm{~d} \theta_{T^{*} N}\right)
$$

The 1-forms $\alpha_{N}^{*} \theta_{T N}$ and $\beta_{N}^{*} \theta_{T^{*} N}$ are not equal, their difference is the differential of a smooth function.

\subsubsection{Lagrangian Submanifolds}

In view of applications to implicit Hamiltonian systems, let us recall here that a Lagrangian submanifold of a symplectic manifold $(M, \omega)$ is a submanifold $N$ whose dimension is half the dimension of $M$, on which the form induced by the symplectic form $\omega$ is 0 .

Let $L: T N \rightarrow \mathbb{R}$ and $H: T^{*} N \rightarrow \mathbb{R}$ be two smooth real valued functions, defined on $T N$ and on $T^{*} N$, respectively. The graphs $\mathrm{d} L(T N)$ and $\mathrm{d} H\left(T^{*} N\right)$ of their differentials are Lagrangian submanifolds of the symplectic manifolds $\left(T^{*} T N, \mathrm{~d} \theta_{T N}\right)$ and $\left(T^{*} T^{*} N, \mathrm{~d} \theta_{T^{*} N}\right)$. Their pull-backs $\alpha_{N}^{-1}(\mathrm{~d} L(T N))$ and $\beta_{N}^{-1}\left(\mathrm{~d} H\left(T^{*} N\right)\right)$ by the symplectomorphisms $\alpha_{N}$ and $\beta_{N}$ are therefore two Lagrangian submanifolds of the manifold $T T^{*} N$ endowed with the symplectic form $\alpha_{N}^{*}\left(\mathrm{~d} \theta_{T N}\right)$, which is equal to the symplectic form $\beta_{N}^{*}\left(\mathrm{~d} \theta_{T^{*} N}\right)$.

The following theorem enlightens some aspects of the relationships between the Hamiltonian and the Lagrangian formalisms. 
Theorem 4 (W. M. Tulczyjew). With the notations specified above Section 4.3.2, let $X_{H}: T^{*} N \rightarrow T T^{*} N$ be the Hamiltonian vector field on the symplectic manifold $\left(T^{*} N, \mathrm{~d} \theta_{N}\right)$ associated to the Hamiltonian $H: T^{*} N \rightarrow \mathbb{R}$, defined by $\mathrm{i}\left(X_{H}\right) \mathrm{d} \theta_{N}=-\mathrm{d} H$. Then

$$
X_{H}\left(T^{*} N\right)=\beta_{N}^{-1}\left(\mathrm{~d} H\left(T^{*} N\right)\right)
$$

Moreover, the equality

$$
\alpha_{N}^{-1}(\mathrm{~d} L(T N))=\beta_{N}^{-1}\left(\mathrm{~d} H\left(T^{*} N\right)\right)
$$

holds if and only if the Lagrangian $L$ is hyper-regular and such that

$$
\mathrm{d} H=\mathrm{d}\left(E_{L} \circ \mathcal{L}_{L}^{-1}\right),
$$

where $\mathcal{L}_{L}: T N \rightarrow T^{*} N$ is the Legendre map and $E_{L}: T N \rightarrow \mathbb{R}$ the energy associated to the Lagrangian $L$.

The interested reader will find the proof of that theorem in the works of Tulczyjew ([34,35]).

When $L$ is not hyper-regular, $\alpha_{N}^{-1}(\mathrm{~d} L(T N))$ still is a Lagrangian submanifold of the symplectic manifold $\left(T T^{*} N, \alpha_{N}^{*}\left(\mathrm{~d} \theta_{T N}\right)\right)$, but it is no more the graph of a smooth vector field $X_{H}$ defined on $T^{*} N$. Tulczyjew proposes to consider this Lagrangian submanifold as an implicit Hamilton equation on $T^{*} N$.

These results can be extended to Lagrangians and Hamiltonians which may depend on time.

\subsection{The Hamiltonian Formalism on Symplectic and Poisson Manifolds}

\subsubsection{The Hamilton Formalism on Symplectic Manifolds}

In pure mathematics as well as in applications of mathematics to mechanics and physics, symplectic manifolds other than cotangent bundles are encountered. A theorem due to the french mathematician Gaston Darboux (1842-1917) asserts that any symplectic manifold $(M, \omega)$ is of even dimension $2 n$ and is locally isomorphic to the cotangent bundle to a $n$-dimensional manifold: in a neighbourhood of each of its point there exist local coordinates $\left(x^{1}, \ldots, x^{n}, p_{1}, \ldots, p_{n}\right)$, called Darboux coordinates with which the symplectic form $\omega$ is expressed exactly as the canonical symplectic form of a cotangent bundle:

$$
\omega=\sum_{i=1}^{n} \mathrm{~d} p_{i} \wedge \mathrm{d} x^{i}
$$

Let $(M, \omega)$ be a symplectic manifold and $H: \mathbb{R} \times M \rightarrow \mathbb{R}$ a smooth function, said to be a time-dependent Hamiltonian. It determines a time-dependent Hamiltonian vector field $X_{H}$ on $M$, such that

$$
\mathrm{i}\left(X_{H}\right) \omega=-\mathrm{d} H_{t},
$$

$H_{t}: M \rightarrow \mathbb{R}$ being the function $H$ in which the variable $t$ is considered as a parameter with respect to which no differentiation is made.

The Hamilton equation determined by $H$ is the differential equation

$$
\frac{\mathrm{d} \psi(t)}{\mathrm{d} t}=X_{H}(t, \psi(t)) .
$$

The Hamiltonian formalism can therefore be applied to any smooth, maybe time dependent Hamiltonian on $M$, even when there is no associated Lagrangian.

The Hamiltonian formalism is not limited to symplectic manifolds: it can be applied, for example, to Poisson manifolds [36], contact manifolds and Jacobi manifolds [37]. For simplicity I will consider only Poisson manifolds. Readers interested in Jacobi manifolds and their generalizations are referred to the papers by Lichnerowicz quoted above and to the very important paper by Kirillov [38]. 
Definition 6. A Poisson manifold is a smooth manifold $P$ whose algebra of smooth functions $C^{\infty}(P, \mathbb{R})$ is endowed with a bilinear composition law, called the Poisson bracket, which associates to any pair $(f, g)$ of smooth functions on $P$ another smooth function denoted by $\{f, g\}$, that composition satisfying the three properties

1. it is skew-symmetric,

$$
\{g, f\}=-\{f, g\}
$$

2. it satisfies the Jacobi identity

$$
\{f,\{g, h\}\}+\{g,\{h, f\}\}+\{h,\{f, g\}\}=0,
$$

3. it satisfies the Leibniz identity

$$
\{f, g h\}=\{f, g\} h+g\{f, h\}
$$

\section{Example 4.}

1. On the vector space of smooth functions defined on a symplectic manifold $(M, \omega)$, there exists a composition law, called the Poisson bracket, which satisfies the properties stated in Definition 6. Let us recall briefly its definition. The symplectic form $\omega$ allows us to associate, to any smooth function $f \in C^{\infty}(M, \mathbb{R})$, a smooth vector field $X_{f} \in A^{1}(M, \mathbb{R})$, called the Hamiltonian vector field associated to $f$, defined by

$$
\mathrm{i}\left(X_{f}\right) \omega=-\mathrm{d} f .
$$

The Poisson bracket $\{f, g\}$ of two smooth functions $f$ and $g \in C^{\infty}(M, \mathbb{R})$ is defined by the three equivalent equalities

$$
\{f, g\}=\mathrm{i}\left(X_{f}\right) \mathrm{d} g=-\mathrm{i}\left(X_{g}\right) \mathrm{d} f=\omega\left(X_{f}, X_{g}\right) .
$$

Any symplectic manifold is therefore a Poisson manifold.

The Poisson bracket of smooth functions defined on a symplectic manifold (when that symplectic manifold is a cotangent bundle) was discovered by Siméon Denis Poisson (1781-1840) [39].

2. Let $\mathcal{G}$ be a finite-dimensional real Lie algebra, and let $\mathcal{G}^{*}$ be its dual vector space. For each smooth function $f \in C^{\infty}\left(\mathcal{G}^{*}, \mathbb{R}\right)$ and each $\zeta \in \mathcal{G}^{*}$, the differential $\mathrm{d} f(\zeta)$ is a linear form on $\mathcal{G}^{*}$, in other words an element of the dual vector space of $\mathcal{G}^{*}$. Identifying with $\mathcal{G}$ the dual vector space of $\mathcal{G}^{*}$, we can therefore consider $\mathrm{d} f(\zeta)$ as an element in $\mathcal{G}$. With this identification, we can define the Poisson bracket of two smooth functions $f$ and $g \in C^{\infty}\left(\mathcal{G}^{*}, \mathbb{R}\right)$ by

$$
\{f, g\}(\zeta)=[\mathrm{d} f(\zeta), \mathrm{d} g(\zeta)], \quad \zeta \in \mathcal{G}^{*},
$$

the bracket in the right hand side being the bracket in the Lie algebra $\mathcal{G}$. The Poisson bracket of functions in $C^{\infty}\left(\mathcal{G}^{*}, \mathbb{R}\right)$ so defined satifies the properties stated in Definition 6. The dual vector space of any finite-dimensional real Lie algebra is therefore endowed with a Poisson structure, called its canonical Lie-Poisson structure or its Kirillov-Kostant-Souriau Poisson structure. Discovered by Sophus Lie, this structure was indeed rediscovered independently by Alexander Kirillov, Bertram Kostant and Jean-Marie Souriau.

3. A symplectic cocycle of a finite-dimensional, real Lie algebra $\mathcal{G}$ is a skew-symmetric bilinear map $\Theta$ : $\mathcal{G} \times \mathcal{G} \rightarrow \mathcal{G}^{*}$ which satisfies, for all $X, Y$ and $Z \in \mathcal{G}$,

$$
\Theta([X, Y], Z)+\Theta([Y, Z], X)+\Theta([Z, X], Y)=0 .
$$

The canonical Lie-Poisson bracket of two smooth functions $f$ and $g \in C^{\infty}\left(\mathcal{G}^{*}, \mathbb{R}\right)$ can be modified by means of the symplectic cocycle $\Theta$, by setting

$$
\{f, g\}_{\Theta}(\zeta)=[\mathrm{d} f(\zeta), \mathrm{d} g(\zeta)]-\Theta(\mathrm{d} f(\zeta), \mathrm{d} g(\zeta)), \quad \zeta \in \mathcal{G}^{*} .
$$


This bracket still satifies the properties stated in Definition 6, therefore defines on $\mathcal{G}^{*}$ a Poisson structure called its canonical Lie-Poisson structure modified by $\Theta$.

\subsubsection{Properties of Poisson Manifolds}

The interested reader will find the proofs of the properties recalled here in [8-11].

1. On a Poisson manifold $P$, the Poisson bracket $\{f, g\}$ of two smooth functions $f$ and $g$ can be expressed by means of a smooth field of bivectors $\Lambda$ :

$$
\{f, g\}=\Lambda(\mathrm{d} f, \mathrm{~d} g), \quad f \text { and } g \in C^{\infty}(P, \mathbb{R}),
$$

called the Poisson bivector field of $P$. The considered Poisson manifold is often denoted by $(P, \Lambda)$. The Poisson bivector field $\Lambda$ identically satisfies

$$
[\Lambda, \Lambda]=0
$$

the bracket $[$,$] in the left hand side being the Schouten-Nijenhuis bracket. That bivector field$ determines a vector bundle morphism $\Lambda^{\sharp}: T^{*} P \rightarrow T P$, defined by

$$
\Lambda(\eta, \zeta)=\left\langle\zeta, \Lambda^{\sharp}(\eta)\right\rangle,
$$

where $\eta$ and $\zeta \in T^{*} P$ are two covectors attached to the same point in $P$.

Readers interested in the Schouten-Nijenhuis bracket will find thorough presentations of its properties in $[40,41]$.

2. Let $(P, \Lambda)$ be a Poisson manifold. A (maybe time-dependent) vector field on $P$ can be associated to each (maybe time-dependent) smooth function $H: \mathbb{R} \times P \rightarrow \mathbb{R}$. It is called the Hamiltonian vector field associated to the Hamiltonian $H$, and denoted by $X_{H}$. Its expression is

$$
X_{H}(t, x)=\Lambda^{\sharp}(x)\left(\mathrm{d} H_{t}(x)\right),
$$

where $\mathrm{d} H_{t}(x)=\mathrm{d} H(t, x)-\frac{\partial H(t, x)}{\partial t} \mathrm{~d} t$ is the differential of the function deduced from $H$ by considering $t$ as a parameter with respect to which no differentiation is made.

The Hamilton equation determined by the (maybe time-dependent) Hamiltonian $H$ is

$$
\frac{\mathrm{d} \varphi(t)}{\mathrm{d} t}=X_{H}\left((t, \varphi(t))=\Lambda^{\sharp}\left(\mathrm{d} H_{t}\right)(\varphi(t)) .\right.
$$

3. Any Poisson manifold is foliated, by a generalized foliation whose leaves may not be all of the same dimension, into immersed connected symplectic manifolds called the symplectic leaves of the Poisson manifold. The value, at any point of a Poisson manifold, of the Poisson bracket of two smooth functions only depends on the restrictions of these functions to the symplectic leaf through the considered point, and can be calculated as the Poisson bracket of functions defined on that leaf, with the Poisson structure associated to the symplectic structure of that leaf. This property was discovered by Alan Weinstein, in his very thorough study of the local structure of Poisson manifolds [42].

\section{Hamiltonian Symmetries}

\subsection{Presymplectic, Symplectic and Poisson Maps and Vector Fields}

Let $M$ be a manifold endowed with some structure, which can be either

- a presymplectic structure, determined by a presymplectic form, i.e., a 2-form $\omega$ which is closed $(\mathrm{d} \omega=0)$, 
- a symplectic structure, determined by a symplectic form $\omega$, i.e., a 2-form $\omega$ which is both closed $(\mathrm{d} \omega=0)$ and nondegenerate $(\operatorname{ker} \omega=\{0\})$,

- a Poisson structure, determined by a smooth Poisson bivector field $\Lambda$ satisfying $[\Lambda, \Lambda]=0$.

Definition 7. A presymplectic (resp. symplectic, resp. Poisson) diffeomorphism of a presymplectic (resp., symplectic, resp. Poisson) manifold $(M, \omega)$ (resp. $(M, \Lambda))$ is a smooth diffeomorphism $f: M \rightarrow M$ such that $f^{*} \omega=\omega\left(\right.$ resp. $\left.f^{*} \Lambda=\Lambda\right)$.

Definition 8. A smooth vector field $X$ on a presymplectic (resp. symplectic, resp. Poisson) manifold $(M, \omega)$ (resp. $(M, \Lambda)$ ) is said to be a presysmplectic (resp. symplectic, resp. Poisson) vector field if $\mathcal{L}(X) \omega=0$ (resp. if $\mathcal{L}(X) \Lambda=0)$, where $\mathcal{L}(X)$ denotes the Lie derivative of forms or mutivector fields with respect to $X$.

Definition 9. Let $(M, \omega)$ be a presymplectic or symplectic manifold. A smooth vector field X on $M$ is said to be Hamiltonian if there exists a smooth function $H: M \rightarrow \mathbb{R}$, called a Hamiltonian for $X$, such that

$$
\mathrm{i}(X) \omega=-\mathrm{d} H .
$$

Not any smooth function on a presymplectic manifold can be a Hamiltonian.

Definition 10. Let $(M, \Lambda)$ be a Poisson manifold. A smooth vector field $X$ on $M$ is said to be Hamiltonian if there exists a smooth function $H \in C^{\infty}(M, \mathbb{R})$, called a Hamiltonian for $X$, such that $X=\Lambda^{\sharp}(\mathrm{d} H)$. An equivalent definition is that

$$
\mathrm{i}(X) \mathrm{d} g=\{H, g\} \quad \text { for any } g \in C^{\infty}(M, \mathbb{R}),
$$

where $\{H, g\}=\Lambda(\mathrm{d} H, \mathrm{~d} g)$ denotes the Poisson bracket of the functions $H$ and $g$.

On a symplectic or a Poisson manifold, any smooth function can be a Hamiltonian.

Proposition 3. A Hamiltonian vector field on a presymplectic (resp. symplectic, resp. Poisson) manifold automatically is a presymplectic (resp. symplectic, resp. Poisson) vector field.

The proof of this result, which is easy, can be found in any book on symplectic and Poisson geoemetry, for example [8-10].

\subsection{Lie Algebras and Lie Groups Actions}

Definition 11. An action on the left (resp. an action on the right) of a Lie group $G$ on a smooth manifold $M$ is a smooth map $\Phi: G \times M \rightarrow M$ (resp. a smooth map $\Psi: M \times G \rightarrow M$ ) such that

- for each fixed $g \in G$, the map $\Phi_{g}: M \rightarrow M$ defined by $\Phi_{g}(x)=\Phi(g, x)$ (resp. the map $\Psi_{g}: M \rightarrow M$ defined by $\Psi_{g}(x)=\Psi(x, g)$ ) is a smooth diffeomorphism of $M$,

- $\Phi_{e}=\operatorname{id}_{M}\left(\right.$ resp. $\left.\Psi_{e}=\operatorname{id}_{M}\right)$, e being the neutral element of $G$,

- for each pair $\left(g_{1}, g_{2}\right) \in G \times G, \Phi_{g_{1}} \circ \Phi_{g_{2}}=\Phi_{g_{1} g_{2}}\left(\right.$ resp. $\Psi_{g_{1}} \circ \Psi_{g_{2}}=\Psi_{g_{2} g_{1}}$ ).

An action of a Lie algebra $\mathcal{G}$ on a smooth manifold $M$ is a Lie algebras morphism of $\mathcal{G}$ into the Lie algebra $A^{1}(M)$ of smooth vector fields on $M$, i.e., a linear map $\psi: \mathcal{G} \rightarrow A^{1}(M)$ which associates to each $X \in \mathcal{G}$ a smooth vector field $\psi(X)$ on $M$ such that for each pair $(X, Y) \in \mathcal{G} \times \mathcal{G}, \psi([X, Y])=[\psi(X), \psi(Y)]$.

Proposition 4. An action $\Psi$, either on the left or on the right, of a Lie group $G$ on a smooth manifold $M$, automatically determines an action $\psi$ of its Lie algebra $\mathcal{G}$ on that manifold, which associates to each $X \in \mathcal{G}$ the vector field $\psi(X)$ on $M$, often denoted by $X_{M}$ and called the fundamental vector field on $M$ associated to $X$. It is defined by

$$
\psi(X)(x)=X_{M}(x)=\left.\frac{\mathrm{d}}{\mathrm{d} s}\left(\Psi_{\exp (s X)}(x)\right)\right|_{s=0}, \quad x \in M,
$$


with the following convention: $\psi$ is a Lie algebras homomorphism when we take for Lie algebra $\mathcal{G}$ of the Lie group $G$ the Lie algebra or right invariant vector fields on $G$ if $\Psi$ is an action on the left, and the Lie algebra of left invariant vector fields on $G$ if $\Psi$ is an action on the right.

Proof. If $\Psi$ is an action of $G$ on $M$ on the left (respectively, on the right), the vector field on $G$ which is right invariant (respectively, left invariant) and whose value at $e$ is $X$, and the associated fundamental vector field $X_{M}$ on $M$, are compatible by the map $g \mapsto \Psi_{g}(x)$. Therefore the map $\psi: \mathcal{G} \rightarrow A^{1}(M)$ is a Lie algebras homomorphism, if we take for definition of the bracket on $\mathcal{G}$ the bracket of right invariant (respectively, left invariant) vector fields on $G$.

Definition 12. When $M$ is a presymplectic (or a symplectic, or a Poisson) manifold, an action $\Psi$ of a Lie group $G$ (respectively, an action $\psi$ of a Lie algebra $\mathcal{G}$ ) on the manifold $M$ is called a presymplectic (or a symplectic, or a Poisson) action if for each $g \in G, \Psi_{g}$ is a presymplectic, or a symplectic, or a Poisson diffeomorphism of $M$ (respectively, if for each $X \in \mathcal{G}, \psi(X)$ is a presymplectic, or a symplectic, or a Poisson vector field on $M$.

Definition 13. An action $\psi$ of a Lie algeba $\mathcal{G}$ on a presymplectic or symplectic manifold $(M, \omega)$, or on a Poisson manifold $(M, \Lambda)$, is said to be Hamiltonian if for each $X \in \mathcal{G}$, the vector field $\psi(X)$ on $M$ is Hamiltonian.

An action $\Psi$ (either on the left or on the right) of a Lie group $G$ on a presymplectic or symplectic manifold $(M, \omega)$, or on a Poisson manifold $(M, \Lambda)$, is said to be Hamiltonian if that action is presymplectic, or symplectic, or Poisson (according to the structure of $M$ ), and if in addition the associated action of the Lie algebra $\mathcal{G}$ of $G$ is Hamiltonian.

Remark 9. A Hamiltonian action of a Lie group, or of a Lie algebra, on a presymplectic, symplectic or Poisson manifold, is automatically a presymplectic, symplectic or Poisson action. This result immediately follows from Proposition 3.

\subsection{Momentum Maps of Hamiltonian Actions}

Proposition 5. Let $\psi$ be a Hamiltonian action of a finite-dimensional Lie algebra $\mathcal{G}$ on a presymplectic, symplectic or Poisson manifold $(M, \omega)$ or $(M, \Lambda)$. There exists a smooth map $J: M \rightarrow \mathcal{G}^{*}$, taking its values in the dual space $\mathcal{G}^{*}$ of the Lie algebra $\mathcal{G}$, such that for each $X \in \mathcal{G}$ the Hamiltonian vector field $\psi(X)$ on $M$ admits as Hamiltonian the function $J_{X}: M \rightarrow \mathbb{R}$, defined by

$$
J_{X}(x)=\langle J(x), X\rangle, \quad x \in M .
$$

The map $J$ is called a momentum map for the Lie algebra action $\psi$. When $\psi$ is the action of the Lie algebra $\mathcal{G}$ of a Lie group $G$ associated to a Hamiltonian action $\Psi$ of a Lie group $G$, J is called a momentum map for the Hamiltonian Lie group action $\Psi$.

The proof of that result, which is easy, can be found for example in [8-10].

Remark 10. The momentum map J is not unique:

- when $(M, \omega)$ is a connected symplectic manifold, J is determined up to addition of an arbitrary constant element in $\mathcal{G}^{*}$;

- when $(M, \Lambda)$ is a connected Poisson manifold, the momentum map $J$ is determined up to addition of an arbitrary $\mathcal{G}^{*}$-valued smooth map which, coupled with any $X \in \mathcal{G}$, yields a Casimir of the Poisson algebra of $(M, \Lambda)$, i.e., a smooth function on $M$ whose Poisson bracket with any other smooth function on that manifold is the function identically equal to 0.

\subsection{Noether's Theorem in Hamiltonian Formalism}

Theorem 5 (Noether's Theorem in Hamiltonian Formalism). Let $X_{f}$ and $X_{g}$ be two Hamiltonian vector fields on a presymplectic or symplectic manifold $(M, \omega)$, or on a Poisson manifold $(M, \Lambda)$, which admit as 
Hamiltonians, respectively, the smooth functions $f$ and $g$ on the manifold $M$. The function $f$ remains constant on each integral curve of $X_{g}$ if and only if $g$ remains constant on each integral curve of $X_{f}$.

Proof. The function $f$ is constant on each integral curve of $X_{g}$ if and only if $\mathrm{i}\left(X_{g}\right) \mathrm{d} f=0$, since each integral curve of $X_{g}$ is connected. We can use the Poisson bracket, even when $M$ is a presymplectic manifold, since the Poisson bracket of two Hamiltonians on a presymplectic manifold still can be defined. So we can write

$$
\mathrm{i}\left(X_{g}\right) \mathrm{d} f=\{g, f\}=-\{f, g\}=-\mathrm{i}\left(X_{f}\right) \mathrm{d} g .
$$

Corollary 2 (of Noether's Theorem in Hamiltonian Formalism). Let $\psi: \mathcal{G} \rightarrow A^{1}(M)$ be a Hamiltonian action of a finite-dimensional Lie algebra $\mathcal{G}$ on a presymplectic or symplectic manifold $(M, \omega)$, or on a Poisson manifold $(M, \Lambda)$, and let $J: M \rightarrow \mathcal{G}^{*}$ be a momentum map of this action. Let $X_{H}$ be a Hamiltonian vector field on $M$ admitting as Hamiltonian a smooth function $H$. If for each $X \in \mathcal{G}$ we have $\mathrm{i}(\psi(X))(\mathrm{d} H)=0$, the momentum map J remains constant on each integral curve of $X_{H}$.

Proof. This result is obtained by applying Theorem 5 to the pairs of Hamiltonian vector fields made by $X_{H}$ and each vector field associated to an element of a basis of $\mathcal{G}$.

\subsection{Symplectic Cocycles}

Theorem 6 (J. M. Souriau [14]). Let $\Phi$ be a Hamiltonian action (either on the left or on the right) of a Lie group $G$ on a connected symplectic manifold $(M, \omega)$ and let $J: M \rightarrow \mathcal{G}^{*}$ be a momentum map of this action. There exists an affine action $A$ (either on the left or on the right) of the Lie group $G$ on the dual $\mathcal{G}^{*}$ of its Lie algebra $\mathcal{G}$ such that the momentum map J is equivariant with respect to the actions $\Phi$ of $G$ on $M$ and $A$ of $G$ on $\mathcal{G}^{*}$, i.e., such that

$$
J \circ \Phi_{g}(x)=A_{g} \circ J(x) \text { for all } g \in G, x \in M \text {. }
$$

The action $A$ can be written, with $g \in G$ and $\xi \in \mathcal{G}^{*}$,

$$
\begin{cases}A(g, \xi)=\operatorname{Ad}_{g^{-1}}^{*}(\xi)+\theta(g) & \text { if } \Phi \text { is an action on the left, } \\ A(\xi, g)=\operatorname{Ad}_{g}^{*}(\xi)-\theta\left(g^{-1}\right) & \text { if } \Phi \text { is an action on the right. }\end{cases}
$$

Proof. Let us assume that $\Phi$ is an action on the left. The fundamental vector field $X_{M}$ associated to each $X \in \mathcal{G}$ is Hamiltonian, with the function $J_{X}: M \rightarrow \mathbb{R}$, given by

$$
J_{X}(x)=\langle J(x), X\rangle, \quad x \in M,
$$

as Hamiltonian. For each $g \in G$ the direct image $\left(\Phi_{g^{-1}}\right)_{*}\left(X_{M}\right)$ of $X_{M}$ by the symplectic diffeomorphism $\Phi_{g^{-1}}$ is Hamiltonian, with $J_{X} \circ \Phi_{g}$ as Hamiltonian. An easy calculation shows that this vector field is the fundamental vector field associated to $\operatorname{Ad}_{g^{-1}}(X) \in \mathcal{G}$. The function

$$
x \mapsto\left\langle J(x), \operatorname{Ad}_{g^{-1}}(X)\right\rangle=\left\langle\operatorname{Ad}_{g^{-1}}^{*} \circ J(x), X\right\rangle
$$

is therefore a Hamiltonian for that vector field. These two functions defined on the connected manifold $M$, which both are admissible Hamiltonians for the same Hamiltonian vector field, differ only by a constant (which may depend on $g \in G$ ). We can set, for any $g \in G$,

$$
\theta(g)=J \circ \Phi_{g}(x)-\operatorname{Ad}_{g^{-1}}^{*} \circ J(x)
$$

and check that the map $A: G \times \mathcal{G}^{*} \rightarrow \mathcal{G}^{*}$ defined in the statement is indeed an action for which $J$ is equivariant.

A similar proof, with some changes of signs, holds when $\Phi$ is an action on the right. 
Proposition 6. Under the assumptions and with the notations of Theorem 6, the map $\theta: G \rightarrow \mathcal{G}^{*}$ is a cocycle of the Lie group $G$ with values in $\mathcal{G}^{*}$, for the coadjoint representation. It means that it satisfies, for all $g$ and $h \in G$,

$$
\theta(g h)=\theta(g)+\operatorname{Ad}_{g^{-1}}^{*}(\theta(h)) .
$$

More precisely $\theta$ is a symplectic cocycle. It means that its differential $T_{e} \theta: T_{e} G \equiv \mathcal{G} \rightarrow \mathcal{G}^{*}$ at the neutral element $e \in G$ can be considered as a skew-symmetric bilinear form on $\mathcal{G}$ :

$$
\Theta(X, Y)=\left\langle T_{e} \theta(X), Y\right\rangle=-\left\langle T_{e} \theta(Y), X\right\rangle .
$$

The skew-symmetric bilinear form $\Theta$ is a symplectic cocycle of the Lie algebra $\mathcal{G}$. It means that it is skew-symmetric and satisfies, for all $X, Y$ and $Z \in \mathcal{G}$,

$$
\Theta([X, Y], Z)+\Theta([Y, Z], X)+\Theta([Z, X], Y)=0 .
$$

Proof. These properties easily follow from the fact that when $\Phi$ is an action on the left, for $g$ and $h \in G$, $\Phi_{g} \circ \Phi_{h}=\Phi_{g h}$ (and a similar equality when $\Phi$ is an action on the right). The interested reader will find more details in $[9,12,14]$.

Proposition 7. Still under the assumptions and with the notations of Theorem 6, the composition law which associates to each pair $(f, g)$ of smooth real-valued functions on $\mathcal{G}^{*}$ the function $\{f, g\}_{\Theta}$ given by

$$
\{f, g\}_{\Theta}(x)=\langle x,[\mathrm{~d} f(x), \mathrm{d} g(x)]\rangle-\Theta(\mathrm{d} f(x), \mathrm{d} g(x)), \quad x \in \mathcal{G}^{*},
$$

$\left(\mathcal{G}\right.$ being identified with its bidual $\mathcal{G}^{* *}$ ), determines a Poisson structure on $\mathcal{G}^{*}$, and the momentum map $J: M \rightarrow \mathcal{G}^{*}$ is a Poisson map, $M$ being endowed with the Poisson structure associated to its symplectic structure.

Proof. The fact that the bracket $(f, g) \mapsto\{f, g\}_{\Theta}$ on $C^{\infty}\left(\mathcal{G}^{*}, \mathbb{R}\right)$ is a Poisson bracket was already indicated in Example 4. It can be verified by easy calculations. The fact that $J$ is a Poisson map can be proven by first looking at linear functions on $\mathcal{G}^{*}$, i.e., elements in $\mathcal{G}$. The reader will find a detailed proof in [12].

Remark 11. When the momentum map $J$ is replaced by another momentum map $J_{1}=J+\mu$, where $\mu \in \mathcal{G}^{*}$ is a constant, the symplectic Lie group cocycle $\theta$ and the symplectic Lie algebra cocycle $\Theta$ are replaced by $\theta_{1}$ and $\Theta_{1}$, respectively, given by

$$
\begin{aligned}
\theta_{1}(g) & =\theta(g)+\mu-\operatorname{Ad}_{g^{-1}}^{*}(\mu), \quad g \in G, \\
\Theta_{1}(X, Y) & =\Theta(X, Y)+\langle\mu,[X, Y]\rangle, \quad X \text { and } Y \in \mathcal{G} .
\end{aligned}
$$

These formulae show that $\theta_{1}-\theta$ and $\Theta_{1}-\Theta$ are symplectic coboundaries of the Lie group $G$ and the Lie algebra $\mathcal{G}$. In other words, the cohomology classes of the cocycles $\theta$ and $\Theta$ only depend on the Hamiltonian action $\Phi$ of $G$ on the symplectic manifold $(M, \omega)$.

\subsection{The Use of Symmetries in Hamiltonian Mechanics}

\subsubsection{Symmetries of the Phase Space}

Hamiltonian Symmetries are often used for the search of solutions of the equations of motion of mechanical systems. The symmetries considered are those of the phase space of the mechanical system. This space is very often a symplectic manifold, either the cotangent bundle to the configuration space with its canonical symplectic structure, or a more general symplectic manifold. Sometimes, after some simplifications, the phase space is a Poisson manifold. 
The Marsden-Weinstein reduction procedure [43,44] or one of its generalizations [10] is the method most often used to facilitate the determination of solutions of the equations of motion. In a first step, a possible value of the momentum map is chosen and the subset of the phase space on which the momentum map takes this value is determined. In a second step, that subset (when it is a smooth manifold) is quotiented by its isotropic foliation. The quotient manifold is a symplectic manifold of a dimension smaller than that of the original phase space, and one has an easier to solve Hamiltonian system on that reduced phase space.

When Hamiltonian symmetries are used for the reduction of the dimension of the phase space of a mechanical system, the symplectic cocycle of the Lie group of symmetries action, or of the Lie algebra of symmetries action, is almost always the zero cocycle.

For example, if the group of symmetries is the canonical lift to the cotangent bundle of a group of symmetries of the configuration space, not only the canonical symplectic form, but the Liouville 1-form of the cotangent bundle itself remains invariant under the action of the symmetry group, and this fact implies that the symplectic cohomology class of the action is zero.

\subsubsection{Symmetries of the Space of Motions}

A completely different way of using symmetries was initiated by Jean-Marie Souriau, who proposed to consider the symmetries of the manifold of motions of the mechanical system. He observed that the Lagrangian and Hamiltonian formalisms, in their usual formulations, involve the choice of a particular reference frame, in which the motion is described. This choice destroys a part of the natural symmetries of the system.

For example, in classical (non-relativistic) mechanics, the natural symmetry group of an isolated mechanical system must contain the symmetry group of the Galilean space-time, called the Galilean group. This group is of dimension 10. It contains not only the group of Euclidean displacements of space which is of dimension 6 and the group of time translations which is of dimension 1, but the group of linear changes of Galilean reference frames which is of dimension 3.

If we use the Lagrangian formalism or the Hamiltonian formalism, the Lagrangian or the Hamiltonian of the system depends on the reference frame: it is not invariant with respect to linear changes of Galilean reference frames.

It may seem strange to consider the set of all possible motions of a system, which is unknown as long as we have not determined all these possible motions. One may ask if it is really useful when we want to determine not all possible motions, but only one motion with prescribed initial data, since that motion is just one point of the (unknown) manifold of motion!

Souriau's answers to this objection are the following.

1. We know that the manifold of motions has a symplectic structure, and very often many things are known about its symmetry properties.

2. In classical (non-relativistic) mechanics, there exists a natural mathematical object which does not depend on the choice of a particular reference frame (even if the decriptions given to that object by different observers depend on the reference frame used by these observers): it is the evolution space of the system.

The knowledge of the equations which govern the system's evolution allows the full mathematical description of the evolution space, even when these equations are not yet solved.

Moreover, the symmetry properties of the evolution space are the same as those of the manifold of motions.

For example, the evolution space of a classical mechanical system with configuration manifold $N$ is

1. in the Lagrangian formalism, the space $\mathbb{R} \times T N$ endowed with the presymplectic form $\mathrm{d} \widehat{\omega}_{L}$, whose kernel is of dimension 1 when the Lagrangian $L$ is hyper-regular,

2. in the Hamiltonian formalism, the space $\mathbb{R} \times T^{*} N$ with the presymplectic form $\mathrm{d} \widehat{\omega}_{H}$, whose kernel too is of dimension 1. 
The Poincaré-Cartan 1-form $\widehat{\omega}_{L}$ in the Lagrangian formalism, or $\widehat{\omega}_{H}$ in the Hamiltonian formalism, depends on the choice of a particular reference frame, made for using the Lagrangian or the Hamiltonian formalism. But their exterior differentials, the presymplectic forms d $\widehat{\omega}_{L}$ or $\mathrm{d} \widehat{\omega}_{H}$, do not depend on that choice, modulo a simple change of variables in the evolution space.

Souriau defined this presymplectic form in a framework more general than those of Lagrangian or Hamiltonian formalisms, and called it the Lagrange form. In this more general setting, it may not be an exact 2-form. Souriau proposed as a new Principle, the assumption that it always projects on the space of motions of the systems as a symplectic form, even in relativistic mechanics in which the definition of an evolution space is not clear. He called this new principle the Maxwell Principle.

Bargmann proved that the symplectic cohomology of the Galilean group is of dimension 1, and Souriau proved that the cohomology class of its action on the manifold of motions of an isolated classical (non-relativistic) mechanical system can be identified with the total mass of the system [14], Chapter III, p. 153.

Readers interested in the Galilean group and momentum maps of its actions are referred to the recent book by de Saxcé and Vallée [45].

\section{Statistical Mechanics and Thermodynamics}

\subsection{Basic Concepts in Statistical Mechanics}

During the XVIII-th and XIX-th centuries, the idea that material bodies (fluids as well as solids) are assemblies of a very large number of small, moving particles, began to be considered by some scientists, notably Daniel Bernoulli (1700-1782), Rudolf Clausius (1822-1888), James Clerk Maxwell (1831-1879) and Ludwig Eduardo Boltzmann (1844-1906), as a reasonable possibility. Attemps were made to explain the nature of some measurable macroscopic quantities (for example the temperature of a material body, the pressure exerted by a gas on the walls of the vessel in which it is contained), and the laws which govern the variations of these macroscopic quantities, by application of the laws of classical mechanics to the motions of these very small particles. Described in the framework of the Hamiltonian formalism, the material body is considered as a Hamiltonian system whose phase space is a very high dimensional symplectic manifold $(M, \omega)$, since an element of that space gives a perfect information about the positions and the velocities of all the particles of the system. The experimental determination of the exact state of the system being impossible, one only can use the probability of presence, at each instant, of the state of the system in various parts of the phase space. Scientists introduced the concept of a statistical state, defined below.

Definition 14. Let $(M, \omega)$ be a symplectic manifold. A statistical state is a probability measure $\mu$ on the manifold $M$.

\subsubsection{The Liouville Measure on a Symplectic Manifold}

On each symplectic manifold $(M, \omega)$, with $\operatorname{dim} M=2 n$, there exists a positive measure $\lambda_{\omega}$, called the Liouville measure. Let us briefly recall its definition. Let $(U, \varphi)$ be a Darboux chart of $(M, \omega)$ Section 4.4.1. The open subset $U$ of $M$ is, by means of the diffeomorphism $\varphi$, identified with an open subset $\varphi(U)$ of $\mathbb{R}^{2 n}$ on which the coordinates (Darboux coordinates) will be denoted by $\left(p_{1}, \ldots, p_{n}, x^{1}, \ldots, x^{n}\right)$. With this identification, the Liouville measure (restricted to $U$ ) is simply the Lebesgue measure on the open subset $\varphi(U)$ of $\mathbb{R}^{2 n}$. In other words, for each Borel subset $A$ of $M$ contained in $U$, we have

$$
\lambda_{\omega}(A)=\int_{\varphi(A)} \mathrm{d} p_{1} \ldots \mathrm{d} p_{n} \mathrm{~d} x^{1} \ldots \mathrm{d} x^{n} .
$$

One can easily check that this definition does not depend on the choice of the Darboux coordinates $\left(p_{1}, \ldots, p_{n}, x^{1}, \ldots, x^{n}\right)$ on $\varphi(A)$. By using an atlas of Darboux charts on $(M, \omega)$, one can easily define $\lambda_{\omega}(A)$ for any Borel subset $A$ of $M$. 
Definition 15. A statistical state $\mu$ on the symplectic manifold $(M, \omega)$ is said to be continuous (respectively, is said to be smooth) if it has a continuous (respectively, a smooth) density with respect to the Liouville measure $\lambda_{\omega}$, i.e., if there exists a continuous function (respectively, a smooth function) $\rho: M \rightarrow \mathbb{R}$ such that, for each Borel subset $A$ of $M$

$$
\mu(A)=\int_{A} \rho \mathrm{d} \lambda_{\omega}
$$

Remark 12. The density $\rho$ of a continuous statistical state on $(M, \omega)$ takes its values in $\mathbb{R}^{+}$and of course satisfies

$$
\int_{M} \rho \mathrm{d} \lambda_{\omega}=1
$$

For simplicity we only consider in what follows continuous, very often even smooth statistical states.

\subsubsection{Variation in Time of a Statistical State}

Let $H$ be a smooth time independent Hamiltonian on a symplectic manifold $(M, \omega), X_{H}$ the associated Hamiltonian vector field and $\Phi^{X_{H}}$ its reduced flow. We consider the mechanical system whose time evolution is described by the flow of $X_{H}$.

If the state of the system at time $t_{0}$, assumed to be perfectly known, is a point $z_{0} \in M$, its state at time $t_{1}$ is the point $z_{1}=\Phi_{t_{1}-t_{0}}^{X_{H}}\left(z_{0}\right)$.

Let us now assume that the state of the system at time $t_{0}$ is not perfectly known, but that a continuous probability measure on the phase space $M$, whose density with respect to the Liouville measure $\lambda_{\omega}$ is $\rho_{0}$, describes the probability distribution of presence of the state of the system at time $t_{0}$. In other words, $\rho_{0}$ is the density of the statistical state of the system at time $t_{0}$. For any other time $t_{1}$, the map $\Phi_{t_{1}-t_{0}}^{X_{H}}$ is a symplectomorphism, therefore leaves invariant the Liouville measure $\lambda_{\omega}$. The probability density $\rho_{1}$ of the statistical state of the system at time $t_{1}$ therefore satisfies, for any $x_{0} \in M$ for which $x_{1}=\Phi_{t_{1}-t_{0}}^{X_{H}}\left(x_{0}\right)$ is defined,

$$
\rho_{1}\left(x_{1}\right)=\rho_{1}\left(\Phi_{t_{1}-t_{0}}^{X_{H}}\left(x_{0}\right)\right)=\rho_{0}\left(x_{0}\right) .
$$

Since $\left(\Phi_{t_{1}-t_{0}}^{X_{H}}\right)^{-1}=\Phi_{t_{0}-t_{1}}^{X_{H}}$, we can write

$$
\rho_{1}=\rho_{0} \circ \Phi_{t_{0}-t_{1}}^{X_{H}} .
$$

Definition 16. Let $\rho$ be the density of a continuous statistical state $\mu$ on the symplectic manifold $(M, \omega)$. The number

$$
s(\rho)=\int_{M} \rho \log \left(\frac{1}{\rho}\right) \mathrm{d} \lambda_{\omega}
$$

is called the entropy of the statistical state $\mu$ or, with a slight abuse of language, the entropy of the density $\rho$.

\section{Remark 13.}

1. By convention we state that $0 \log 0=0$. With that convention the function $x \mapsto x \log x$ is continuous on $\mathbb{R}^{+}$. If the integral on the right hand side of the equality which defines $s(\rho)$ does not converge, we state that $s(\rho)=-\infty$. With these conventions, $s(\rho)$ exists for any continuous probability density $\rho$.

2. The above Definition 16 of the entropy of a statistical state, founded on ideas developed by Boltzmann in his Kinetic Theory of Gases [46], specially in the derivation of his famous (and controversed) Theorem $\hat{E} t a$, is too related with the ideas of Claude Shannon [47] on information theory. The use of information theory in thermodynamics was more recently proposed by Jaynes [48,49] and Mackey [18]. For a very nice discussion of the use of probability concepts in physics and application of information theory in quantum mechanics, the reader is referred to the paper by Balian [50]. 
The entropy $s(\rho)$ of a probability density $\rho$ has very remarkable variational properties discussed in the following definitions and proposition.

Definition 17. Let $\rho$ be the density of a smooth statistical state on a symplectic manifold $(M, \omega)$.

1. For each function $f$ defined on $M$, taking its values in $\mathbb{R}$ or in some finite-dimensional vector space, such that the integral on the right hand side of the equality

$$
\mathcal{E}_{\rho}(f)=\int_{M} f \rho \mathrm{d} \lambda_{\omega}
$$

converges, the value $\mathcal{E}_{\rho}(f)$ of that integral is called the mean value of $f$ with respect to $\rho$.

2. Let $f$ be a smooth function on $M$, taking its values in $\mathbb{R}$ or in some finite-dimensional vector space, satisfying the properties stated above. A smooth infinitesimal variation of $\rho$ with fixed mean value of $f$ is a smooth map, defined on the product $]-\varepsilon, \varepsilon\left[\times M\right.$, with values in $\mathbb{R}^{+}$, where $\varepsilon>0$,

$$
(\tau, z) \mapsto \rho(\tau, z), \quad \tau \in]-\varepsilon, \varepsilon[, z \in M,
$$

such that

- for $\tau=0$ and any $z \in M, \rho(0, z)=\rho(z)$,

- for each $\tau \in]-\varepsilon, \varepsilon\left[, z \mapsto \rho_{\tau}(z)=\rho(\tau, z)\right.$ is a smooth probability density on $M$ such that

$$
\mathcal{E}_{\rho_{\tau}}(f)=\int_{M} \rho_{\tau} f \mathrm{~d} \lambda_{\omega}=\mathcal{E}_{\rho}(f) .
$$

3. The entropy function $s$ is said to be stationary at the probability density $\rho$ with respect to smooth infinitesimal variations of $\rho$ with fixed mean value of $f$, if for any smooth infinitesimal variation $(\tau, z) \mapsto \rho(\tau, z)$ of $\rho$ with fixed mean value of $f$

$$
\left.\frac{\mathrm{d} s\left(\rho_{\tau}\right)}{\mathrm{d} \tau}\right|_{\tau=0}=0
$$

Proposition 8. Let $H: M \rightarrow \mathbb{R}$ be a smooth Hamiltonian on a symplectic manifold $(M, \omega)$ and $\rho$ be the density of a smooth statistical state on $M$ such that the integral defining the mean value $\mathcal{E}_{\rho}(H)$ of $H$ with respect to $\rho$ converges. The entropy function $s$ is stationary at $\rho$ with respect to smooth infinitesimal variations of $\rho$ with fixed mean value of $H$, if and only if there exists a real $b \in \mathbb{R}$ such that, for all $z \in M$,

$$
\rho(z)=\frac{1}{P(b)} \exp (-b H(z)), \text { with } P(b)=\int_{M} \exp (-b H) \mathrm{d} \lambda_{\omega} .
$$

Proof. Let $\tau \mapsto \rho_{\tau}$ be a smooth infinitesimal variation of $\rho$ with fixed mean value of $H$. Since $\int_{M} \rho_{\tau} \mathrm{d} \lambda_{\omega}$ and $\int_{M} \rho_{\tau} H \mathrm{~d} \lambda_{\omega}$ do not depend on $\tau$, it satisfies, for all $\left.\tau \in\right]-\varepsilon, \varepsilon[$,

$$
\int_{M} \frac{\partial \rho(\tau, z)}{\partial \tau} \mathrm{d} \lambda_{\omega}(z)=0, \int_{M} \frac{\partial \rho(\tau, z)}{\partial \tau} H(z) \mathrm{d} \lambda_{\omega}(z)=0 .
$$

Moreover an easy calculation leads to

$$
\left.\frac{\mathrm{d} s\left(\rho_{\tau}\right)}{\mathrm{d} \tau}\right|_{\tau=0}=-\left.\int_{M} \frac{\partial \rho(\tau, z)}{\partial \tau}\right|_{\tau=0}\left(1+\log (\rho(z)) \mathrm{d} \lambda_{\omega}(z) .\right.
$$

A well known result in calculus of variations shows that the entropy function $s$ is stationary at $\rho$ with respect to smooth infinitesimal variations of $\rho$ with fixed mean value of $H$, if and only if there exist two real constants $a$ and $b$, called Lagrange multipliers, such that, for all $z \in M$,

$$
1+\log (\rho)+a+b H=0,
$$


which leads to

$$
\rho=\exp (-1-a-b H) .
$$

By writing that $\int_{M} \rho \mathrm{d} \lambda_{\omega}=1$, we see that $a$ is determined by $b$ :

$$
\exp (1+a)=P(b)=\int_{M} \exp (-b H) \mathrm{d} \lambda_{\omega} .
$$

Definition 18. Let $H: M \rightarrow \mathbb{R}$ be a smooth Hamiltonian on a symplectic manifold $(M, \omega)$. For each $b \in \mathbb{R}$ such that the integral on the right side of the equality

$$
P(b)=\int_{M} \exp (-b H) \mathrm{d} \lambda_{\omega}
$$

converges, the smooth probability measure on $M$ with density (with respect to the Liouville measure)

$$
\rho(b)=\frac{1}{P(b)} \exp (-b H)
$$

is called the Gibbs statistical state associated to $b$. The function $P: b \mapsto P(b)$ is called the partition function.

The following proposition shows that the entropy function, not only is stationary at any Gibbs statistical state, but in a certain sense attains at that state a strict maximum.

Proposition 9. Let $H: M \rightarrow \mathbb{R}$ be a smooth Hamiltonian on a symplectic manifold $(M, \omega)$ and $b \in \mathbb{R}$ be such that the integral defining the value $P(b)$ of the partition function $P$ at $b$ converges. Let

$$
\rho_{b}=\frac{1}{P(b)} \exp (-b H)
$$

be the probability density of the Gibbs statistical state associated to $b$. We assume that the Hamiltonian $H$ is bounded by below, i.e., that there exists a constant $m$ such that $m \leq H(z)$ for any $z \in M$. Then the integral defining

$$
\mathcal{E}_{\rho_{b}}(H)=\int_{M} \rho_{b} H \mathrm{~d} \lambda_{\omega}
$$

converges. For any other smooth probability density $\rho_{1}$ such that

$$
\mathcal{E}_{\rho_{1}}(H)=\mathcal{E}_{\rho_{b}}(H),
$$

we have

$$
s\left(\rho_{1}\right) \leq s\left(\rho_{b}\right),
$$

and the equality $s\left(\rho_{1}\right)=s\left(\rho_{b}\right)$ holds if and only if $\rho_{1}=\rho_{b}$.

Proof. Since $m \leq H$, the function $\rho_{b} \exp (-b H)$ satisfies $0 \leq \rho_{b} \exp (-b H) \leq \exp (-m b) \rho_{b}$, therefore is integrable on $M$. Let $\rho_{1}$ be any smooth probability density on $M$ satisfying $\mathcal{E}_{\rho_{1}}(H)=\mathcal{E}_{\rho_{b}}(H)$. The function defined on $\mathbb{R}^{+}$

$$
x \mapsto h(x)= \begin{cases}x \log \left(\frac{1}{x}\right) & \text { if } x>0 \\ 0 & \text { if } x=0\end{cases}
$$

being convex, its graph is below the tangent at any of its points $\left(x_{0}, h\left(x_{0}\right)\right)$. We therefore have, for all $x>0$ and $x_{0}>0$,

$$
h(x) \leq h\left(x_{0}\right)-\left(1+\log x_{0}\right)\left(x-x_{0}\right)=x_{0}-x\left(1+\log x_{0}\right) .
$$


With $x=\rho_{1}(z)$ and $x_{0}=\rho_{b}(z), z$ being any element in $M$, that inequality becomes

$$
h\left(\rho_{1}(z)\right)=\rho_{1}(z) \log \left(\frac{1}{\rho_{1}(z)}\right) \leq \rho_{b}(z)-\left(1+\log \rho_{b}(z)\right) \rho_{1}(z) .
$$

By integration over $M$, using the fact that $\rho_{b}$ is the probability density of the Gibbs state associated to $b$, we obtain

$$
s\left(\rho_{1}\right) \leq 1-1-\int_{M} \rho_{1} \log \rho_{b} \mathrm{~d} \lambda_{\omega}=s\left(\rho_{b}\right) .
$$

We have proven the inequality $s\left(\rho_{1}\right) \leq s\left(\rho_{b}\right)$. If $\rho_{1}=\rho_{b}$, we have of course the equality $s\left(\rho_{1}\right)=s\left(\rho_{b}\right)$. Conversely if $s\left(\rho_{1}\right)=s\left(\rho_{b}\right)$, the functions defined on $M$

$$
z \mapsto \varphi_{1}(z)=\rho_{1}(z) \log \left(\frac{1}{\rho_{1}(z)}\right) \quad \text { and } \quad z \mapsto \varphi(z)=\rho_{b}(z)-\left(1+\log \rho_{b}(z)\right) \rho_{1}(z)
$$

are continuous on $M$ except, maybe, for $\varphi$, at points $z$ at which $\rho_{b}(z)=0$ and $\rho_{1}(z) \neq 0$, but the set of such points is of measure 0 since $\varphi$ is integrable. They satisfy the inequality $\varphi_{1} \leq \varphi$. Both are integrable on $M$ and have the same integral. The function $\varphi-\varphi_{1}$ is everywhere $\geq 0$, is integrable on $M$ and its integral is 0 . That function is therefore everywhere equal to 0 on $M$. We can write, for any $z \in M$,

$$
\rho_{1}(z) \log \left(\frac{1}{\rho_{1}(z)}\right)=\rho_{b}(z)-\left(1+\log \rho_{b}(z)\right) \rho_{1}(z) .
$$

For each $z \in M$ such that $\rho_{1}(z) \neq 0$, we can divide that equality by $\rho_{1}(z)$. We obtain

$$
\frac{\rho_{b}(z)}{\rho_{1}(z)}-\log \left(\frac{\rho_{b}(z)}{\rho_{1}(z)}\right)=1 .
$$

Since the function $x \mapsto x-\log x$ reaches its minimum, equal to 1 , for a unique value of $x>0$, that value being 1 , we see that for each $z \in M$ at which $\rho_{1}(z)>0$, we have $\rho_{1}(z)=\rho_{b}(z)$. At points $z \in M$ at which $\rho_{1}(z)=0$, Equation (6) shows that $\rho_{b}(z)=0$. Therefore $\rho_{1}=\rho_{b}$.

Remark 14. The maximality property of the entropy function $\rho \mapsto s(\rho)$ at a Gibbs state density $\rho_{b}$ proven in Proposition 9 of course implies the stationarity of that function at $\rho_{b}$ with respect to smooth infinitesimal variations of $\rho$ with fixed mean value of $H$, proven in Proposition 8. That Proposition therefore could be omitted. We chose to keep it because its proof is much easier than that of Proposition 9, and explains why it is interesting to look at probability densities proportional to $\exp (-b H)$ for some $b \in \mathbb{R}$.

The following proposition shows that a Gibbs statistical state remains invariant under the flow of the Hamiltonian vector field $X_{H}$. One can therefore say that a Gibbs state is a statistical equilibrium state. Of course there exist statistical equilibrium states other than Gibbs states.

Proposition 10. Let $H$ be a smooth Hamiltonian bounded by below on a symplectic manifold $(M, \omega), b \in \mathbb{R}$ be such that the integral defining the value $P(b)$ of the partition function $P$ at $b$ converges. The Gibbs state associated to $b$ remains invariant under the flow of of the Hamiltonian vector field $X_{H}$.

Proof. The density $\rho_{b}$ of the Gibbs state associated to $b$, with respect to the Liouville measure $\lambda_{\omega}$, is

$$
\rho_{b}=\frac{1}{P(b)} \exp (-b H)
$$

Since $H$ is constant along each integral curve of $X_{H}, \rho_{b}$ too is constant along each integral curve of $X_{H}$. Moreover, the Liouville measure $\lambda_{\omega}$ remains invariant under the flow of $X_{H}$. Therefore the Gibbs probability measure associated to $b$ too remains invariant under that flow. 


\subsection{Thermodynamic Equilibria and Thermodynamic Functions}

\subsubsection{Assumptions Made in this Section.}

Any Hamiltonian $H$ defined on a symplectic manifold $(M, \omega)$ considered in this section will be assumed to be smooth, bounded by below and such that for any real $b>0$, each one of the three functions, defined on $M, z \mapsto \exp (-b H(z)), z \mapsto|H(z)| \exp (-b H(z))$ and $z \mapsto$ $(H(z))^{2} \exp (-b H(z))$ is everywhere smaller than some function defined on $M$ integrable with respect to the Liouville measure $\lambda_{\omega}$. The integrals which define

$$
P(b)=\int_{M} \exp (-b H) \mathrm{d} \lambda_{\omega} \quad \text { and } \quad \mathcal{E}_{\rho_{b}}(H)=\int_{M} H \exp (-b H) \mathrm{d} \lambda_{\omega}
$$

therefore converge.

Proposition 11. Let $H$ be a Hamiltonian defined on a symplectic manifold $(M, \omega)$ satisfying the assumptions indicated in Section 6.2.1. For any real $b>0$ let

$$
P(b)=\int_{M} \exp (-b H) \mathrm{d} \lambda_{\omega} \quad \text { and } \quad \rho_{b}=\frac{1}{P(b)} \exp (-b H)
$$

be the value at $b$ of the partition function $P$ and the probability density of the Gibbs statistical state associated to $b$, and

$$
E(b)=\mathcal{E}_{\rho_{b}}(H)=\frac{1}{P(b)} \int_{M} H \exp (-b H) \mathrm{d} \lambda_{\omega}
$$

be the mean value of $H$ with respect to the probability density $\rho_{b}$. The first and second derivatives with respect to $b$ of the partition function $P$ exist, are continuous functions of $b$ given by

$$
\frac{\mathrm{d} P(b)}{\mathrm{d} b}=-P(b) E(b), \quad \frac{\mathrm{d}^{2} P(b)}{\mathrm{d} b^{2}}=\int_{M} H^{2} \exp (-b H) \mathrm{d} \lambda_{\omega}=P(b) \mathcal{E}_{\rho_{b}}\left(H^{2}\right) .
$$

The derivative with respect to $b$ of the function E exists and is a continuous function of $b$ given by

$$
\frac{\mathrm{d} E(b)}{\mathrm{d} b}=-\frac{1}{P(b)} \int_{M}\left(H-\mathcal{E}_{\rho_{b}}(H)\right)^{2} \mathrm{~d} \lambda_{\omega}=-\mathcal{E}_{\rho_{b}}\left(\left(H-\mathcal{E}_{\rho_{b}}(H)\right)^{2}\right) .
$$

Let $S(b)$ be the entropy $s\left(\rho_{b}\right)$ of the Gibbs statistical state associated to $b$. The function $S$ can be expressed in terms of $P$ and $E$ as

$$
S(b)=\log (P(b))+b E(b) .
$$

Its derivative with respect to $b$ exists and is a continuous function of $b$ given by

$$
\frac{\mathrm{d} S(b)}{\mathrm{d} b}=b \frac{\mathrm{d} E(b)}{\mathrm{d} b}
$$

Proof. Using the assumptions Section 6.2.1, we see that the functions $b \mapsto P(b)$ and $b \mapsto \mathcal{E}_{\rho_{b}}(H)=E(b)$, defined by integrals on $M$, have a derivative with respect to $b$ which is continuous and which can be calculated by derivation under the sign $\int_{M}$. The indicated results easily follow, if we observe that for any function $f$ on $M$ such that $\mathcal{E}_{\rho_{b}}(f)$ and $\mathcal{E}_{\rho_{b}}\left(f^{2}\right)$ exist, we have the formula, well known in Probability theory,

$$
\mathcal{E}_{\rho_{b}}\left(f^{2}\right)-\left(\mathcal{E}_{\rho_{b}}(f)\right)^{2}=\mathcal{E}_{\rho_{b}}\left(\left(f-\mathcal{E}_{\rho_{b}}(f)\right)^{2}\right)
$$

\subsubsection{Physical Meaning of the Introduced Functions}

Let us consider a physical system, for example a gas contained in a vessel bounded by rigid, thermally insulated walls, at rest in a Galilean reference frame. We assume that its evolution can 
be mathematically described by means of a Hamiltonian system on a symplectic manifold $(M, \omega)$ whose Hamiltonian $H$ satisfies the assumptions Section 6.2.1. For physicists, a Gibbs statistical state, i.e., a probability measure of density $\rho_{b}=\frac{1}{P(b)} \exp (-b H)$ on $M$, is a thermodynamic equilibrium of the physical system. The set of possible thermodynamic equilibria of the system is therefore indexed by a real parameter $b>0$. The following argument will show what physical meaning can have that parameter.

Let us consider two similar physical systems, mathematically described by two Hamiltonian systems, of Hamiltonians $H_{1}$ on the symplectic manifold $\left(M_{1}, \omega_{1}\right)$ and $H_{2}$ on the symplectic manifold $\left(M_{2}, \omega_{2}\right)$. We first assume that they are independent and both in thermodynamic equilibrium, with different values $b_{1}$ and $b_{2}$ of the parameter $b$. We denote by $E_{1}\left(b_{1}\right)$ and $E_{2}\left(b_{2}\right)$ the mean values of $H_{1}$ on the manifold $M_{1}$ with respect to the Gibbs state of density $\rho_{1, b_{1}}$ and of $H_{2}$ on the manifold $M_{2}$ with respect to the Gibbs state of density $\rho_{2, b_{2}}$. We assume now that the two systems are coupled in a way allowing an exchange of energy. For example, the two vessels containing the two gases can be separated by a wall allowing a heat transfer between them. Coupled together, they make a new physical system, mathematically described by a Hamiltonian system on the symplectic manifold $\left(M_{1} \times M_{2}, p_{1}^{*} \omega_{1}+p_{2}^{*} \omega_{2}\right)$, where $p_{1}: M_{1} \times M_{2} \rightarrow M_{1}$ and $p_{2}: M_{1} \times M_{2} \rightarrow M_{2}$ are the canonical projections. The Hamiltonian of this new system can be made as close to $H_{1} \circ p_{1}+H_{2} \circ p_{2}$ as one wishes, by making very small the coupling between the two systems. The mean value of the Hamiltonian of the new system is therefore very close to $E_{1}\left(b_{1}\right)+E_{2}\left(b_{2}\right)$. When the total system will reach a state of thermodynamic equilibrium, the probability densities of the Gibbs states of its two parts, $\rho_{1, b^{\prime}}$ on $M_{1}$ and $\rho_{2, b^{\prime}}$ on $M_{2}$ will be indexed by the same real number $b^{\prime}>0$, which must be such that

$$
E_{1}\left(b^{\prime}\right)+E_{2}\left(b^{\prime}\right)=E_{1}\left(b_{1}\right)+E_{2}\left(b_{2}\right) .
$$

By Proposition 11, we have, for all $b>0$,

$$
\frac{\mathrm{d} E_{1}(b)}{\mathrm{d} b} \leq 0, \quad \frac{\mathrm{d} E_{2}(b)}{\mathrm{d} b} \leq 0 .
$$

Therefore $b^{\prime}$ must lie between $b_{1}$ and $b_{2}$. If, for example, $b_{1}<b_{2}$, we see that $E_{1}\left(b^{\prime}\right) \leq E_{1}\left(b_{1}\right)$ and $E_{2}\left(b^{\prime}\right) \geq E_{2}\left(b_{2}\right)$. In order to reach a state of thermodynamic equilibrium, energy must be transferred from the part of the system where $b$ has the smallest value, towards the part of the system where $b$ has the highest value, until, at thermodynamic equilibrium, $b$ has the same value everywhere. Everyday experience shows that thermal energy flows from parts of a system where the temperature is higher, towards parts where it is lower. For this reason physicists consider the real variable $b$ as a way to appreciate the temperature of a physical system in a state of thermodynamic equilibrium. More precisely, they state that

$$
b=\frac{1}{k T}
$$

where $T$ is the absolute temperature and $k$ a constant depending on the choice of units of energy and temperature, called Boltzmann's constant in honour of the great Austrian scientist Ludwig Eduard Boltzmann (1844-1906).

For a physical system mathematically described by a Hamiltonian system on a symplectic manifold $(M, \omega)$, with $H$ as Hamiltonian, in a state of thermodynamic equilibrium, $E(b)$ and $S(b)$ are the internal energy and the entropy of the system.

\subsubsection{Towards Thermodynamic Equilibrium}

Everyday experience shows that a physical system, when submitted to external conditions which remain unchanged for a sufficiently long time, very often reaches a state of thermodynamic equilibrium. At first look, it seems that Lagrangian or Hamiltonian systems with time-independent Lagrangians or Hamiltonians cannot exhibit a similar behaviour. Let us indeed consider a mechanical system whose 
configuration space is a smooth manifold $N$, described in the Lagrangian formalism by a smooth time-independent hyper-regular Lagarangian $L: T N \rightarrow \mathbb{R}$ or, in the Hamiltonian formalism, by the associated Hamiltonian $H_{L}: T^{*} N \rightarrow \mathbb{R}$. Let $t \mapsto \overrightarrow{x(t)}$ be a motion of that system, $\overrightarrow{x_{0}}=\overrightarrow{x\left(t_{0}\right)}$ and $\overrightarrow{x_{1}}=\overrightarrow{x\left(t_{0}\right)}$ be the configurations of the system for that motion at times $t_{0}$ and $t_{1}$. There exists another motion $t \mapsto \overrightarrow{x^{\prime}(t)}$ of the system for which $\overrightarrow{x^{\prime}\left(t_{0}\right)}=\overrightarrow{x_{1}}$ and $\overrightarrow{x^{\prime}\left(t_{1}\right)}=\overrightarrow{x_{0}}$ : since the equations of motion are invariant by time reversal, the motion $t \mapsto \overrightarrow{x^{\prime}(t)}$ is obtained simply by taking as initial condition at time $t_{0} \overrightarrow{x^{\prime}\left(t_{0}\right)}=\overrightarrow{x\left(t_{1}\right)}$ and $\left.\frac{\mathrm{d} \overrightarrow{x^{\prime}(t)}}{\mathrm{d} t}\right|_{t=t_{0}}=-\left.\frac{\mathrm{d} \overrightarrow{x(t)}}{\mathrm{d} t}\right|_{t=t_{1}}$. Another more serious argument against a kind of thermodynamic behaviour of Lagarangian or Hamiltonian systems rests on the famous recurrence theorem due to Poincaré [51]. This theorem asserts indeed that when the useful part of the phase space of the system is of a finite total measure, almost all points in an arbitrarily small open subset of the phase space are recurrent, i.e., the motion starting of such a point at time $t_{0}$ repeatedly crosses that open subset again and again, infinitely many times when $t \rightarrow+\infty$.

Let us now consider, instead of perfectly defined states, i.e., points in phase space, statistical states, and ask the question: When at time $t=t_{0}$ a Hamiltonian system on a symplectic manifold $(M, \omega)$ is in a statistical state given by some probability measure of density $\rho_{0}$ with respect to the Liouville measure $\lambda_{\omega}$, does its statistical state converge, when $t \rightarrow+\infty$, towards the probability measure of a Gibbs state? This question should be made more precise by specifying what physical meaning has a statistical state and in what mathematical sense a statistical state can converge towards the probability measure of a Gibbs state. A positive partial answer was given by Ludwig Boltzmann when, developing his kinetic theory of gases, he proved his famous (but controversed) Êta theorem stating that the entropy of the statistical state of a gas of small particles is a monotonously increasing function of time. This question, linked with time irreversibility in physics, is still the subject of important researches, both by physicists and by mathematicians. The reader is referred to the paper [50] by Balian for a more thorough discussion of that question.

\subsection{Examples of Thermodynamic Equilibria}

\subsubsection{Classical Monoatomic Ideal Gas}

In classical mechanics, a dilute gas contained in a vessel at rest in a Galilean reference frame is mathematically described by a Hamiltonian system made by a large number of very small massive particles, which interact by very brief collisions between themselves or with the walls of the vessel, whose motions between two collisions are free. Let us first assume that these particles are material points and that no external field is acting on them, other than that describing the interactions by collisions with the walls of the vessel.

The Hamiltonian of one particle in a part of the phase space in which its motion is free is simply

$$
\frac{1}{2 m}\|\vec{p}\|^{2}=\frac{1}{2 m}\left(p_{1}^{2}+p_{2}^{2}+p_{3}^{2}\right), \text { with } \vec{p}=m \vec{v},
$$

where $m$ is the mass of the particle, $\vec{v}$ its velocity vector and $\vec{p}$ its linear momentum vector (in the considered Galilean reference frame), $p_{1}, p_{2}$ and $p_{3}$ the components of $\vec{p}$ in a fixed orhtonormal basis of the physical space.

Let $N$ be the total number of particles, which may not have all the same mass. We use a integer $i \in\{1,2, \ldots, N\}$ to label the particles and denote by $m_{i}, \overrightarrow{x_{i}}, \overrightarrow{v_{i}}, \overrightarrow{p_{i}}$ the mass and the vectors position, velocity and linear momentum of the $i$-th particle.

The Hamiltonian of the gas is therefore

$$
H=\sum_{i=1}^{N} \frac{1}{2 m_{i}}\left\|\overrightarrow{p_{i}}\right\|^{2}+\text { terms involving the collisions between particles and with the walls. }
$$


Interactions of the particles with the walls of the vessel are essential for allowing the motions of particles to remain confined. Interactions between particles are essential to allow the exchanges between them of energy and momentum, which play an important part in the evolution with time of the statistical state of the system. However it appears that while these terms are very important to determine the system's evolution with time, they can be neglected, when the gas is dilute enough, if we only want to determine the final statistical state of the system, once a thermodynamic equilibrium is established. The Hamiltonian used will therefore be

$$
H=\sum_{i=1}^{N} \frac{1}{2 m_{i}}\left\|\overrightarrow{p_{i}}\right\|^{2}
$$

The partition function is

$$
P(b)=\int_{M} \exp (-b H) \mathrm{d} \lambda_{\omega}=\int_{D} \exp \left(-b \sum_{i=1}^{N} \frac{1}{2 m_{i}}\left\|\vec{p}_{i}\right\|^{2}\right) \prod_{i=1}^{N}\left(\mathrm{~d} \overrightarrow{x_{i}} \mathrm{~d} \overrightarrow{p_{i}}\right),
$$

where $D$ is the domain of the $6 N$-dimensional space spanned by the position vectors $\overrightarrow{x_{i}}$ and linear momentum vectors $\overrightarrow{p_{i}}$ of the particles in which all the $\overrightarrow{x_{i}}$ lie within the vessel containing the gas. An easy calculation leads to

$$
P(b)=V^{N}\left(\frac{2 \pi}{b}\right)^{3 N / 2} \prod_{i=1}^{N}\left(m_{i}^{3 / 2}\right)=\prod_{i=1}^{N}\left[V\left(\frac{2 \pi m_{i}}{b}\right)^{3 / 2}\right],
$$

where $V$ is the volume of the vessel which contains the gas. The probability density of the Gibbs state associated to $b$, with respect to the Liouville measure, therefore is

$$
\rho_{b}=\prod_{i=1}^{N}\left[\frac{1}{V}\left(\frac{b}{2 \pi m_{i}}\right)^{3 / 2} \exp \left(\frac{-b\left\|\overrightarrow{p_{i}}\right\|^{2}}{2 m_{i}}\right)\right] .
$$

We observe that $\rho_{b}$ is the product of the probability densities $\rho_{i, b}$ for the $i$-th particle

$$
\rho_{i, b}=\frac{1}{V}\left(\frac{b}{2 \pi m_{i}}\right)^{3 / 2} \exp \left(\frac{-b\left\|\overrightarrow{p_{i}}\right\|^{2}}{2 m_{i}}\right)
$$

The $2 N$ stochastic vectors $\overrightarrow{x_{i}}$ and $\overrightarrow{p_{i}}, i=1, \ldots, N$ are therefore independent. The position $\overrightarrow{x_{i}}$ of the $i$-th particle is uniformly distributed in the volume of the vessel, while the probability measure of its linear momentum $\overrightarrow{p_{i}}$ is the classical Maxwell-Boltzmann probability distribution of linear momentum for an ideal gas of particles of mass $m_{i}$, first obtained by Maxwell in 1860. Moreover we see that the three components $p_{i 1}, p_{i 2}$ and $p_{i 3}$ of the linear momentum $\overrightarrow{p_{i}}$ in an orhonormal basis of the physical space are independent stochastic variables.

By using the formulae given in Proposition 11 the internal energy $E(b)$ and the entropy $S(b)$ of the gas can be easily deduced from the partition function $P(b)$. Their expressions are

$$
E(b)=\frac{3 N}{2 b}, \quad S(b)=\frac{3}{2} \sum_{i=1}^{N} \log m_{i}+\left(\frac{3}{2}(1+\log (2 \pi))+\log V\right) N-\frac{3 N}{2} \log b .
$$

We see that each of the $N$ particles present in the gas has the same contribution $\frac{3}{2 b}$ to the internal energy $E(b)$, which does not depend on the mass of the particle. Even more: each degree of freedom of each particle, i.e., each of the the three components of the the linear momentum of the particle on the three axes of an orthonormal basis, has the same contribution $\frac{1}{2 b}$ to the internal energy $E(b)$. This result is known in physics under the name Theorem of equipartition of the energy at a thermodynamic equilibrium. It can be easily generalized for polyatomic gases, in which a particle may carry, in addition 
to the kinetic energy due to the velocity of its centre of mass, a kinetic energy due to the particle's rotation around its centre of mass. The reader can consult the books by Souriau [14] and Mackey [18] where the kinetic theory of polyatomic gases is discussed.

The pressure in the gas, denoted by $\Pi(b)$ because the notation $P(b)$ is already used for the partition function, is due to the change of linear momentum of the particles which occurs at a collision of the particle with the walls of the vessel containing the gas (or with a probe used to measure that pressure). A classical argument in the kinetic theory of gases (see for example [52,53]) leads to

$$
\Pi(b)=\frac{2}{3} \frac{E(b)}{V}=\frac{N}{V b} .
$$

This formula is the well known equation of state of an ideal monoatomic gas relating the number of particles by unit of volume, the pressure and the temperature.

With $b=\frac{1}{k T}$, the above expressions are exactly those used in classical thermodynamics for an ideal monoatomic gas.

\subsubsection{Classical Ideal Monoatomic Gas in a Gravity Field}

Let us now assume that the gas, contained in a cylindrical vessel of section $\Sigma$ and length $h$, with a vertical axis, is submitted to the vertical gravity field of intensity $g$ directed downwards. We choose Cartesian coordinates $x, y, z$, the $z$ axis being vertical directed upwards, the bottom of the vessel being in the horizontal surface $z=0$. The Hamiltonian of a free particle of mass $m$, position and linear momentum vectors $\vec{x}$ (components $x, y, z$ ) and $\vec{p}$ (components $p_{x}, p_{y}$ and $p_{z}$ ) is

$$
\frac{1}{2 m}\left(p_{x}^{2}+p_{y}^{2}+p_{z}^{2}\right)+m g z .
$$

As in the previous section we neglect the parts of the Hamiltonian of the gas corresponding to collisions between the particles, or between a particle and the walls of the vessel. The Hamiltonian of the gas is therefore

$$
H=\sum_{i=1}^{N}\left(\frac{1}{2 m_{i}}\left(p_{i x}^{2}+p_{i y}^{2}+p_{i z}^{2}\right)+m_{i} g z_{i}\right) .
$$

Calculations similar to those of the previous section lead to

$$
\begin{aligned}
P(b) & =\prod_{i=1}^{N}\left[\Sigma\left(\frac{2 \pi m_{i}}{b}\right)^{3 / 2} \frac{1-\exp \left(-m_{i} g b h\right)}{m_{i} g b}\right], \\
\rho_{b} & =\frac{1}{P(b)} \exp \left[-b \sum_{i=1}^{N}\left(\frac{\left\|\overrightarrow{p_{i}}\right\|^{2}}{2 m_{i}}+m_{i} g z_{i}\right)\right] .
\end{aligned}
$$

The expression of $\rho_{b}$ shows that the $2 N$ stochastic vectors $\overrightarrow{x_{i}}$ and $\overrightarrow{p_{i}}$ still are independent, and that for each $i \in\{1, \ldots, N\}$, the probability law of each stochastic vector $\overrightarrow{p_{i}}$ is the same as in the absence of gravity, for the same value of $b$. Each stochastic vector $\overrightarrow{x_{i}}$ is no more uniformly distributed in the vessel containing the gas: its probability density is higher at lower altitudes $z$, and this nonuniformity is more important for the heavier particles than for the lighter ones.

As in the previous section, the formulae given in Proposition 11 allow the calculation of $E(b)$ and $S(b)$. We observe that $E(b)$ now includes the potential energy of the gas in the gravity field, therefore should no more be called the internal energy of the gas.

\subsubsection{Relativistic Monoatomic Ideal Gas}

In a Galilean reference frame, we consider a relativistic point particle of rest mass $m$, moving at a velocity $\vec{v}$. We denote by $v$ the modulus of $\vec{v}$ and by $c$ the modulus of the velocity of light. 
The motion of the particle can be mathematically described by means of the Euler-Lagrange equations, with the Lagrangian

$$
L=-m c^{2} \sqrt{1-\frac{v^{2}}{c^{2}}}
$$

The components of the linear momentum $\vec{p}$ of the particle, in an orthonormal frame at rest in the considered Galilean reference frame, are

$$
p_{i}=\frac{\partial L}{\partial v^{i}}=\frac{m v^{i}}{\sqrt{1-\frac{v^{2}}{c^{2}}}}, \text { therefore } \vec{p}=\frac{m \vec{v}}{\sqrt{1-\frac{v^{2}}{c^{2}}}} .
$$

Denoting by $p$ the modulus of $\vec{p}$, the Hamiltonian of the particle is

$$
H=\vec{p} \cdot \vec{v}-L=\frac{m c^{2}}{\sqrt{1-\frac{v^{2}}{c^{2}}}}=c \sqrt{p^{2}+m^{2} c^{2}} .
$$

Let us consider a relativistic gas, made of $N$ point particles indexed by $i \in\{1, \ldots, N\}, m_{i}$ being the rest mass of the $i$-th particle. With the same assumptions as those made in Section 6.3.1, we can take for Hamiltonian of the gas

$$
H=c \sum_{i=1}^{N} \sqrt{p_{i}^{2}+m^{2} c^{2}} .
$$

With the same notations as those of Section 6.3.1, the partition function $P$ of the gas takes the value, for each $b>0$,

$$
P(b)=\int_{D} \exp \left(-b c \sum_{i=1}^{N} \sqrt{\left(p_{i}\right)^{2}+m^{2} c^{2}}\right) \prod_{i=1}^{N}\left(\mathrm{~d} \overrightarrow{x_{i}} \mathrm{~d} \overrightarrow{p_{i}}\right) .
$$

This integral can be expressed in terms of the Bessel function $K_{2}$, whose expression is, for each $x>0$,

$$
K_{2}(x)=x \int_{0}^{+\infty} \exp (-x \operatorname{ch} \chi) \operatorname{sh}^{2} \chi \operatorname{ch} \chi \mathrm{d} \chi
$$

We have

$$
\begin{aligned}
P(b) & =\left(\frac{4 \pi V c}{b}\right)^{N} \prod_{i=1}^{N}\left(m_{i}^{2} K_{2}\left(m_{i} b c^{2}\right)\right) \\
\rho_{b} & =\frac{1}{P(b)} \exp \left(-b c \sum_{i=1}^{N} \sqrt{p_{i}{ }^{2}+m_{i}^{2} c^{2}}\right) .
\end{aligned}
$$

This probability density of the Gibbs state shows that the $2 N$ stochastic vectors $\overrightarrow{x_{i}}$ and $\overrightarrow{p_{i}}$ are independent, that each $\overrightarrow{x_{i}}$ is uniformly distributed in the vessel containing the gas and that the probability density of each $\overrightarrow{p_{i}}$ is exactly the probability distribution of the linear momentum of particles in a relativistic gas called the Maxwell-Jüttner distribution, obtained by Ferencz Jüttner (1878-1958) in 1911, discussed in the book by the Irish mathematician and physicist Synge [54].

Of course, the formulae given in Proposition 11 allow the calculation of the internal energy $E(b)$, the entropy $S(b)$ and the pressure $\Pi(b)$ of the relativistic gas.

\subsubsection{Relativistic IDeal Gas of Massless Particles}

We have seen in the previous Chapter that in an inertial reference frame, the Hamiltonian of a relativistic point particle of rest mass $m$ is $c \sqrt{p^{2}+m^{2} c^{2}}$, where $p$ is the modulus of the linear momentum vector $\vec{p}$ of the particle in the considered reference frame. This expression 
still has a meaning when the rest mass $m$ of the particle is 0 . In an orthonormal reference frame, the equations of motion of a particle whose motion is mathematically described by a Hamiltonian system with Hamiltonian

$$
H=c p=c \sqrt{p_{1}^{2}+p_{2}^{2}+p_{3}^{2}}
$$

are

$$
\left\{\begin{array}{l}
\frac{\mathrm{d} x^{i}}{\mathrm{~d} t}=\frac{\partial H}{\partial p_{i}}=c \frac{p_{i}}{p} \quad(1 \leq i \leq 3), \\
\frac{\mathrm{d} p_{i}}{\mathrm{~d} t}=-\frac{\partial H}{\partial x^{i}}=0,
\end{array}\right.
$$

which shows that the particle moves on a straight line at the velocity of light $c$. It seems therefore reasonable to describe a gas of $N$ photons in a vessel of volume $V$ at rest in an inertial reference frame by a Hamiltonian system, with the Hamiltonian

$$
H=c \sum_{i=1}^{N}\left\|\overrightarrow{p_{i}}\right\|=c \sum_{i=1}^{N} \sqrt{p_{i 1^{2}}+p_{i 2}^{2}+p_{i 3}^{2}} .
$$

With the same notations as those used in the previous section, the partition function $P$ of the gas takes the value, for each $b>0$,

$$
P(b)=\int_{D} \exp \left(-b c \sum_{i=1}^{N}\left\|\overrightarrow{p_{i}}\right\|\right) \prod_{i=1}^{N}\left(\mathrm{~d} \overrightarrow{x_{i}} \mathrm{~d} \overrightarrow{p_{i}}\right)=\left(\frac{8 \pi V}{c^{3} b^{3}}\right)^{N} .
$$

The probability density of the corresponding Gibbs state, with respect to the Liouville measure $\lambda_{\omega}=\prod_{i=1}^{N}\left(\mathrm{~d} \overrightarrow{x_{i}} \mathrm{~d} \overrightarrow{p_{i}}\right)$, is

$$
\rho_{b}=\prod_{i=1}^{N}\left(\frac{c^{3} b^{3}}{8 \pi V}\right) \exp \left(-b c\left\|\overrightarrow{p_{i}}\right\|\right) .
$$

This formula appears in the books by Synge [54] and Souriau [14]. Physicists consider it as not adequate for the description of a gas of photons contained in a vessel at thermal equilibrium because the number of photons in the vessel, at any given temperature, cannot be imposed: it results from the processes of absorption and emission of photons by the walls of the vessel, heated at the imposed temperature, which spontaneously occur. In other words, this number is a stochastic function whose probability law is imposed by Nature. Souriau proposes, in his book [14], a way to account for the possible variation of the number of photons. Instead of using the phase space of the system of $N$ massless relativistic particles contained in a vessel, he uses the manifold of motions $M_{N}$ of that system (which is symplectomorphic to its phase space). He considers that the manifold of motions $M$ of a system of photons in the vessel is the disjoint union

$$
M=\bigcup_{N \in \mathbb{N}} M_{N},
$$

of all the manifolds of motions $M_{N}$ of a system of $N$ massless relativistic particles in the vessel, for all possible values of $N \in \mathbb{N}$. Fo $N=0$ the manifold $M_{0}$ is reduced to a singleton with, as Liouville measure, the measure which takes the value 1 on the only non empty part of that manifold (the whole manifold $M_{0}$ ). Moreover, since any photon cannot be distinguished from any other photon, two motions of the system with the same number $N$ of massless particles which only differ by the labelling of these particles must be considered as identical. Souriau considers too that since the number $N$ of photons freely adjusts itself, the value of the parameter $b=\frac{1}{k T}$ must, at thermodynamic equilibrium, be the same in all parts $M_{N}$ of the system, $N \in \mathbb{N}$. He uses too the fact that a photon can have two different states of (circular) polarization. With these assumptions the value at any $b$ of the partition function of the system is 


$$
P(b)=\sum_{N=0}^{+\infty} \frac{1}{N !}\left(\frac{16 \pi V}{c^{3} b^{3}}\right)^{N}=\exp \left(\frac{16 \pi V}{c^{3} b^{3}}\right) .
$$

The number $N$ of photons in the vessel at thermodynamic equilibrium is a stochastic function which takes the value $n$ with the probability

$$
\text { Probability }([N=n])=\frac{1}{n !}\left(\frac{16 \pi V}{c^{3} b^{3}}\right)^{n} \exp \left(-\frac{16 \pi V}{c^{3} b^{3}}\right) .
$$

The expression of the partition function $P$ allows the calculation of the internal energy, the entropy and all other thermodynamic functions of the system. However, the formula so obtained for the distribution of photons of various energies at a given temperature does not agree with the law, in very good agreement with experiments, obtained by Max Planck (1858-1947) in 1900. An assembly of photons in thermodynamic equilibrium evidently cannot be described as a classical Hamiltonian system. This fact played an important part for the development of quantum mechanics.

\subsubsection{Specific Heat of Solids}

The motion of a one-dimensional harmonic oscillator can be described by a Hamiltonian system with, as Hamiltonian,

$$
H(p, q)=\frac{p^{2}}{2 m}+\frac{\mu q^{2}}{2} .
$$

The idea that the heat energy of a solid comes from the small vibrations, at a microscopic scale, of its constitutive atoms, lead physicists to attempt to mathematically describe a solid as an assembly of a large number $N$ of three-dimensional harmonic oscillators. By dealing separately with each proper oscillation mode, the solid can even be described as an assembly of $3 \mathrm{~N}$ one-dimensional harmonic oscillators. Exanges of energy between these oscillators is allowed by the existence of small couplings between them. However, for the determination of the thermodynamic equilibria of the solid we will, as in the previous section for ideal gases, consider as negligible the energy of interactions between the oscillators. We therefore take for Hamiltonian of the solid

$$
H=\sum_{i=1}^{3 N}\left(\frac{p_{i}^{2}}{2 m_{i}}+\frac{\mu_{i} q_{i}^{2}}{2}\right) \text {. }
$$

The value of the paritition function $P$, for any $b>0$, is

$$
P(b)=\int_{\mathbb{R}^{6 N}} \exp \left[-b \sum_{i=1}^{3 N}\left(\frac{p_{i}{ }^{2}}{2 m_{i}}+\frac{\mu_{i} q_{i}{ }^{2}}{2}\right)\right] \prod_{i=1}^{3 N}\left(\mathrm{~d} p_{i} \mathrm{~d} q_{i}\right)=\prod_{i=1}^{3 N}\left(\frac{1}{v_{i}}\right) b^{-3 N},
$$

where

$$
v_{i}=\frac{1}{2 \pi} \sqrt{\frac{\mu_{i}}{m_{i}}}
$$

is the frequency of the $i$-th harmonic oscillator.

The internal energy of the solid is

$$
E(b)=-\frac{\mathrm{d} \log P(b)}{\mathrm{d} b}=\frac{3 N}{b} .
$$

We observe that it only depends on the the temperature and on the number of atoms in the solid, not on the frequencies $v_{i}$ of the harmonic oscillators. With $b=\frac{1}{k T}$ this result is in agreement with the empirical law for the specific heat of solids, in good agreement with experiments at high temperature, discovered in 1819 by the French scientists Pierre Louis Dulong (1785-1838) and Alexis Thérèse Petit (1791-1820). 


\section{Generalization for Hamiltonian Actions}

\subsection{Generalized Gibbs States}

In his book [15] and in several papers [13,16,17], Souriau extends the concept of a Gibbs state for a Hamiltonian action of a Lie group $G$ on a symplectic manifold $(M, \omega)$. Usual Gibbs states defined in Section 6 for a smooth Hamiltonian $H$ on a symplectic manifold $(M, \omega)$ appear as special cases, in which the Lie group is a one-parameter group. If the symplectic manifold $(M, \omega)$ is the phase space of the Hamiltonian system, that one-parameter group, whose parameter is the time $t$, is the group of evolution, as a function of time, of the state of the system, starting from its state at some arbitrarily chosen initial time $t_{0}$. If $(M, \omega)$ is the symplectic manifold of all the motions of the system, that one-parameter group, whose parameter is a real $\tau \in \mathbb{R}$, is the transformation group which maps one motion of the system with some initial state at time $t_{0}$ onto the motion of the system with the same initial state at another time $\left(t_{0}+\tau\right)$. We discuss below this generalization.

Notations and Conventions

In this section, $\Phi: G \times M \rightarrow M$ is a Hamiltonian action (for example on the left) of a Lie group $G$ on a symplectic manifold $(M, \omega)$. We denote by $\mathcal{G}$ the Lie algebra of $G$, by $\mathcal{G}^{*}$ its dual space and by $J: M \rightarrow \mathcal{G}^{*}$ a momentum map of the action $\Phi$.

Definition 19. Let $b \in \mathcal{G}$ be such that the integrals on the right hand sides of the equalities

$$
\begin{aligned}
P(b) & =\int_{M} \exp (-\langle J, b\rangle) \mathrm{d} \lambda_{\omega} \text { and } \\
E_{J}(b) & =\mathcal{E}_{\rho_{b}}(J)=\frac{1}{P(b)} \int_{M} J \exp (-\langle J, b\rangle) \mathrm{d} \lambda_{\omega}
\end{aligned}
$$

converge. The smooth probability measure on $M$ with density (with respect to the Liouville measure $\lambda_{\omega}$ on $M$ )

$$
\rho_{b}=\frac{1}{P(b)} \exp (-\langle J, b\rangle)
$$

is called the generalized Gibbs statistical state associated to $b$. The functions $b \mapsto P(b)$ and $b \mapsto E_{J}(b)$ so defined on the subset of $\mathcal{G}$ made by elements $b$ for which the integrals defining $P(b)$ and $E_{J}(b)$ converge are called the partition function associated to the momentum map $J$ and the mean value of $J$ at generalized Gibbs states.

The following Proposition generalizes 9.

Proposition 12. Let $b \in \mathcal{G}$ be such that the integrals defining $P(b)$ and $E_{J}(b)$ in Definition 19 converge, and $\rho_{b}$ be the density of the generalized Gibbs state associated to $b$. The entropy $s\left(\rho_{b}\right)$, which will be denoted by $S(b)$, exists and is given by

$$
S(b)=\log (P(b))+\left\langle E_{J}(b), b\right\rangle=\log (P(b))-\langle D(\log P(b)), b\rangle .
$$

Moreover, for any other smooth probability density $\rho_{1}$ such that

$$
\mathcal{E}_{\rho_{1}}(J)=\mathcal{E}_{\rho_{b}}(J)=E_{J}(b),
$$

we have

$$
s\left(\rho_{1}\right) \leq s\left(\rho_{b}\right),
$$

and the equality $s\left(\rho_{1}\right)=s\left(\rho_{b}\right)$ holds if and only if $\rho_{1}=\rho_{b}$. 
Proof. Equation (7) follows from $\log \left(\frac{1}{\rho_{b}}\right)=\log (P(b))+\langle J, b\rangle$, and $D(\log P(b))=-E_{J}(b)$. The remaining of the proof is the same as that of Proposition 9.

\section{Remark 15.}

1. The second part of Equation (7), $S(b)=\log (P(b))-\langle D(\log P(b)), b\rangle$, expresses the fact that the functions $\log (P(b))$ and $-S(b)$ are Legendre transforms of each other: they are linked by the same relation as the relation which links a smooth Lagrangian $L$ and the associated energy $E_{L}$.

2. The Liouville measure $\lambda_{\omega}$ remains invariant under the Hamiltonian action $\Phi$, since the symplectic form $\omega$ itself remains invariant under that action. However, we have not a full analogue of Proposition 10 because the momentum map $J$ does not remain invariant under the action $\Phi$. We only have the partial anologue stated below.

3. Legendre transforms were used by Massieu in thermodynamics in his very early works [55,56], more systematically presented in [57], in which he introduced his characteristic functions (today called thermodynamic potentials) allowing the determination of all the thermodynamic functions of a physical system by partial derivations of a suitably chosen characteristic function. For a modern presentation of that subject the reader is referred to [58,59], Chapter 5, pp. 131-152.

Proposition 13. Let $b \in \mathcal{G}$ be such that the integrals defining $P(b)$ and $E_{J}(b)$ in Definition 19 converge. The generalized Gibbs state associated to $b$ remains invariant under the restriction of the Hamiltonian action $\Phi$ to the one-parameter subgroup of $G$ generated by $b,\{\exp (\tau b) \mid \tau \in \mathbb{R}\}$.

Proof. The orbits of the action on $M$ of the subgroup $\{\exp (\tau b) \mid \tau \in \mathbb{R}\}$ of $G$ are the integral curves of the Hamiltonian vector field whose Hamiltonian is $\langle J, b\rangle$, which of course is constant on each of these curves. Therefore the proof of Proposition 10 is valid for that subgroup.

\subsection{Generalized Thermodynamic Functions}

Assumptions Made in this Section

Notations and conventions being the same as in Section 7.1, let $\Omega$ be the largest open subset of the Lie algebra $\mathcal{G}$ of $G$ containing all $b \in \mathcal{G}$ satisfying the following properties:

- the functions defined on $M$, with values, respectively, in $\mathbb{R}$ and in the dual $\mathcal{G}^{*}$ of $\mathcal{G}$,

$$
z \mapsto \exp (-\langle J(z), b\rangle) \text { and } z \mapsto J(z) \exp (-\langle J(z), b\rangle)
$$

are integrable on $M$ with respect to the Liouville measure $\lambda_{\omega}$;

- moreover their integrals are differentiable with respect to $b$, their differentials are continuous and can be calculated by differentiation under the $\operatorname{sign} \int_{M}$.

It is assumed in this section that the considered Hamiltonian action $\Phi$ of the Lie group $G$ on the symplectic manifold $(M, \omega)$ and its momentum map $J$ are such that the open subset $\Omega$ of $\mathcal{G}$ is not empty. This condition is not always satisfied when $(M, \omega)$ is a cotangent bundle, but of course it is satisfied when it is a compact manifold.

Proposition 14. Let $\Phi: G \times M \rightarrow M$ be a Hamiltonian action of a Lie group $G$ on a symplectic manifold $(M, \omega)$ satisfying the assumptions indicated in Section 7.2. The partition function P associated to the momentum map J and the mean value $E_{J}$ of J for generalized Gibbs states Definition 19 are defined and continuously differentiable on the open subset $\Omega$ of $\mathcal{G}$. For each $b \in \Omega$, the differentials at $b$ of the functions $P$ and $\log P$ (which are linear maps defined on $\mathcal{G}$, with values in $\mathbb{R}$, in other words elements of $\mathcal{G}^{*}$ ) are given by

$$
D P(b)=-P(b) E_{J}(b), \quad D(\log P)(b)=-E_{J}(b) .
$$


For each $b \in \Omega$, the differential at $b$ of the map $E_{J}$ (which is a linear map defined on $\mathcal{G}$, with values in its dual $\mathcal{G}^{*}$ ) is given by

$$
\left\langle D E_{J}(b)(Y), Z\right\rangle=\left\langle E_{J}(b), Y\right\rangle\left\langle E_{J}(b), Z\right\rangle-\mathcal{E}_{\rho_{b}}(\langle J, Y\rangle\langle J, Z\rangle), \quad \text { with } Y \text { and } Z \in \mathcal{G},
$$

where we have written, as in Definition 17,

$$
\mathcal{E}_{\rho_{b}}(\langle J, Y\rangle\langle J, Z\rangle)=\frac{1}{P(b)} \int_{M}\langle J, Y\rangle\langle J, Z\rangle \exp (-\langle J, b\rangle) \mathrm{d} \lambda_{\omega} .
$$

At each $b \in \Omega$, the differential of the entropy function $S$ Proposition 12, which is a linear map defined on $\mathcal{G}$, with values in $\mathbb{R}$, in other words an element of $\mathcal{G}^{*}$, is given by

$$
\langle D S(b), Y\rangle=\left\langle D E_{J}(b)(Y), b\right\rangle, \quad Y \in \mathcal{G} .
$$

Proof. By assumptions Section 7.2, the differentials of $P$ and $E_{J}$ can be calculated by differentiation under the sign $\int_{M}$. Easy (but tedious) calculations lead to the indicated results.

Corollary 3. With the same assumptions and notations as those in Proposition 14 , for any $b \in \Omega$ and $Y \in \mathcal{G}$,

$$
\left\langle D E_{J}(b)(Y), Y\right\rangle=-\frac{1}{P(b)} \int_{M}\left\langle J-E_{J}(b), Y\right\rangle^{2} \mathrm{~d} \lambda_{\omega} \leq 0 .
$$

Proof. This result follows from the well known result in Probability theory already used in the proof of Proposition 11.

The momentum map J of the Hamiltonian action $\Phi$ is not uniquely determined: for any constant $\mu \in \mathcal{G}^{*}, J_{1}=J+\mu$ too is a momentum map for $\Phi$. The following proposition indicates how the generalized thermodynamic functions $P, E_{J}$ and $S$ change when $J$ is replaced by $J_{1}$.

Proposition 15. With the same assumptions and notations as those in Proposition 14 , let $\mu \in \mathcal{G}^{*}$ be a constant. When the momentum map $J$ is replaced by $J_{1}=J+\mu$, the open subset $\Omega$ of $\mathcal{G}$ remains unchanged, while the generalized thermodynamic functions $P, E_{J}$ and $S$, are replaced, respectively, by $P_{1}, E_{J_{1}}$ and $S_{1}$, given by

$$
P_{1}(b)=\exp (-\langle\mu, b\rangle) P(b), \quad E_{J_{1}}(b)=E_{J}(b)+\mu, \quad S_{1}(b)=S(b) .
$$

The Gibbs satistical state and its density $\rho_{b}$ with respect to the Liouville measure $\lambda_{\omega}$ remain unchanged.

Proof. We have

$$
\exp (-\langle J+\mu, b\rangle)=\exp (-\langle\mu, b\rangle) \exp (-\langle J, b\rangle) .
$$

The indicated results follow by easy calculations.

The following proposition indicates how the generalized thermodynamic functions $P, E_{J}$ and $S$ vary along orbits of the adjoint action of the Lie group $G$ on its Lie algebra $\mathcal{G}$.

Proposition 16. The assumptions and notations are the same as those in Proposition 14. The open subset $\Omega$ of $\mathcal{G}$ is an union of orbits of the adjoint action of $G$ on $\mathcal{G}$. In other words, for each $b \in \Omega$ and each $g \in G$, $\operatorname{Ad}_{g} b \in \Omega$. Moreover, let $\theta: G \rightarrow \mathcal{G}^{*}$ be the symplectic cocycle of $G$ for the coadjoin action of $G$ on $\mathcal{G}^{*}$ such that, for any $g \in G$,

$$
J \circ \Phi_{g}=\operatorname{Ad}_{g^{-1}}^{*} \circ J+\theta(g)
$$


Then for each $b \in \Omega$ and each $g \in G$

$$
\begin{aligned}
P\left(\operatorname{Ad}_{g} b\right) & =\exp \left(\left\langle\theta\left(g^{-1}\right), b\right\rangle\right) P(b)=\exp \left(-\left\langle\operatorname{Ad}_{g}^{*} \theta(g), b\right\rangle\right) P(b), \\
E_{J}\left(\operatorname{Ad}_{g} b\right) & =\operatorname{Ad}_{g^{-1}}^{*} E_{J}(b)+\theta(g), \\
S\left(\operatorname{Ad}_{g} b\right) & =S(b) .
\end{aligned}
$$

Proof. We have

$$
\begin{aligned}
P\left(\operatorname{Ad}_{g} b\right) & =\int_{M} \exp \left(-\left\langle J, \operatorname{Ad}_{g} b\right\rangle\right) \mathrm{d} \lambda_{\omega}=\int_{M} \exp \left(-\left\langle\operatorname{Ad}_{g}^{*} J, b\right\rangle\right) \mathrm{d} \lambda_{\omega} \\
& =\int_{M} \exp \left(-\left\langle J \circ \Phi_{g^{-1}}-\theta\left(g^{-1}, b\right\rangle\right) \mathrm{d} \lambda_{\omega}\right. \\
& =\exp \left(\left\langle\theta\left(g^{-1}\right), b\right\rangle\right) P(b)=\exp \left(-\left\langle\operatorname{Ad}_{g}^{*} \theta(g), b\right\rangle\right) P(b),
\end{aligned}
$$

since $\theta\left(g^{-1}\right)=-\operatorname{Ad}_{g}^{*} \theta(g)$. By using Propositions 14 and 12, the other results easily follow.

Remark 16. The equality

$$
E_{J}\left(\operatorname{Ad}_{g} b\right)=\operatorname{Ad}_{g^{-1}}^{*} E_{J}(b)+\theta(g)
$$

means that the map $E_{J}: \Omega \rightarrow \mathcal{G}^{*}$ is equivariant with respect to the adjoint action of $G$ on the open subset $\Omega$ of its Lie algebra $\mathcal{G}$ and its affine action on the left on $\mathcal{G}^{*}$

$$
(g, \xi) \mapsto \operatorname{Ad}_{g^{-1}}^{*} \xi+\theta(g), \quad g \in G, \quad \xi \in \mathcal{G}^{*} .
$$

Proposition 17. The assumptions and notations are the same as those in Proposition 14. For each $b \in \Omega$ and each $X \in \mathcal{G}$, we have

$$
\begin{aligned}
\left\langle E_{J}(b),[X, b]\right\rangle & =\langle\Theta(X), b\rangle, \\
D E_{J}(b)([X, b]) & =-\operatorname{ad}_{X}^{*} E_{J}(b)+\Theta(X),
\end{aligned}
$$

where $\Theta=T_{e} \theta: \mathcal{G} \rightarrow \mathcal{G}^{*}$ is the 1-cocycle of the Lie algebra $\mathcal{G}$ associated to the 1-cocycle $\theta$ of the Lie group $G$.

Proof. Let us set $g=\exp (\tau X)$ in the first equality in Proposition 16, derive that equality with respect to $\tau$, and evaluate the result at $\tau=0$. We obtain

$$
D P(b)([X, b])=-P(b)\langle\Theta(X), b\rangle .
$$

Since, by the first equality of Proposition $14, D P(b)=-P(b) E_{J}(b)$, the first stated equality follows.

Let us now set $g=\exp (\tau X)$ in the second equality in Proposition 16, derive that equality with respect to $\tau$, and evaluate the result at $\tau=0$. We obtain the second equality stated.

Corollary 4. With the assumptions and notations of Proposition 17, let us define, for each $b \in \Omega$, a linear map $\Theta_{b}: \mathcal{G} \rightarrow \mathcal{G}^{*}$ by setting

$$
\Theta_{b}(X)=\Theta(X)-\operatorname{ad}_{X}^{*} E_{J}(b) .
$$

The map $\Theta_{b}$ is a symplectic 1-cocycle of the Lie algebra $\mathcal{G}$ for the coadjoint representation, which satisfies

$$
\Theta_{b}(b)=0 .
$$

Moreover if we replace the momentum map $J$ by $J_{1}=J+\mu$, with $\mu \in \mathcal{G}^{*}$ constant, the 1-cocycle $\Theta_{b}$ remains unchanged. 
Proof. For $X, Y$ and $Z$ in $\mathcal{G}$, we have since $\Theta$ is a 1-cocycle, $\sum_{\operatorname{circ}(X, Y, Z)}$ meaning a sum over circular permutations of $X, Y$ and $Z$, using the Jacobi identity in $\mathcal{G}$, we have

$$
\begin{aligned}
\sum_{\operatorname{circ}(X, Y, Z)}\left\langle\Theta_{b}(X),[Y, Z]\right\rangle & =\sum_{\operatorname{circ}(X, Y, Z)}\left\langle-\operatorname{ad}_{X}^{*} E_{J}(b),[Y, Z]\right\rangle \\
& =\sum_{\operatorname{circ}(X, Y, Z)}\left\langle-E_{J}(b),[X,[Y, Z]]\right\rangle \\
& =0 .
\end{aligned}
$$

The linear map $\Theta_{b}$ is therefore a 1 cocycle, even a symplectic 1-cocycle since for all $X$ and $Y \in \mathcal{G}$, $\left\langle\Theta_{b}(X), Y\right\rangle=-\left\langle\Theta_{b}(Y), X\right\rangle$.

Using the first equality stated in Proposition 17, we have for any $X \in \mathcal{G}$

$$
\left\langle\Theta_{b}(b), X\right\rangle=\left\langle\Theta(b)-\operatorname{ad}_{b}^{*} E_{J}(b), X\right\rangle=-\langle\Theta(X), b\rangle+\left\langle E_{J}(b),[X, b]\right\rangle=0 .
$$

If we replace $J$ by $J_{1}=J+\mu$, the map $X \mapsto \Theta(X)$ is replaced by $X \mapsto \Theta_{1}(X)=\Theta(X)+\operatorname{ad}_{X}^{*} \mu$ and $E_{J}(b)$ by $E_{J_{1}}(b)=E_{J}(b)+\mu$, therefore $\Theta_{b}$ remains unchanged.

The following lemma will allow us to define, for each $b \in \Omega$, a remarkable symmetric bilinear form on the vector subspace $[b, \mathcal{G}]=\{[b, X] ; X \in \mathcal{G}\}$ of the Lie algebra $\mathcal{G}$.

Lemma 1. Let $\Xi$ be a 1-cocycle of a finite-dimensional Lie algebra $\mathcal{G}$ for the coadjoint representation. For each $b \in \operatorname{ker} \Xi$, let $F_{b}=[\mathcal{G}, b]$ be the set of elements $X \in \mathcal{G}$ which can be written $X=\left[X_{1}, b\right]$ for some $X_{1} \in \mathcal{G}$. Then $F_{b}$ is a vector subspace of $\mathcal{G}$, and the value of the right hand side of the equality

$$
\Gamma_{b}(X, Y)=\left\langle\Xi\left(X_{1}\right), Y\right\rangle, \text { with } X_{1} \in \mathcal{G}, X=\left[X_{1}, b\right] \in F_{b}, Y \in F_{b},
$$

depends only on $X$ and $Y$, not on the choice of $X_{1} \in \mathcal{G}$ such that $X=\left[X_{1}, b\right]$. That equality defines a bilinear form $\Gamma_{b}$ on $F_{b}$ which is symmetric, i.e., satisfies

$$
\Gamma_{b}(X, Y)=\Gamma_{b}(Y, X) \text { for all } X \text { and } Y \in F_{b} .
$$

Proof. Let $X_{1}$ and $X_{1}^{\prime} \in \mathcal{G}$ be such that $\left[X_{1}, b\right]=\left[X_{1}^{\prime}, b\right]=X$. Let $Y_{1} \in \mathcal{G}$ be such that $\left[Y_{1}, b\right]=Y$. We have

$$
\begin{aligned}
\left\langle\Xi\left(X_{1}-X_{1}^{\prime}\right), Y\right\rangle & =\left\langle\Xi\left(X_{1}-X_{1}^{\prime}\right),\left[Y_{1}, b\right]\right\rangle \\
& =-\left\langle\Xi\left(Y_{1}\right),\left[b, X_{1}-X_{1}^{\prime}\right]\right\rangle-\left\langle\Xi(b),\left[X_{1}-X_{1}^{\prime}, Y_{1}\right]\right\rangle \\
& =0
\end{aligned}
$$

since $\Xi(b)=0$ and $\left[b, X_{1}-X_{1}^{\prime}\right]=0$. We have shown that $\left\langle\Xi\left(X_{1}\right), Y\right\rangle=\left\langle\Xi\left(X_{1}^{\prime}\right), Y\right\rangle$. Therefore $\Gamma_{b}$ is a bilinear form on $F_{b}$. Similarly

$$
\left\langle\Xi\left(X_{1}\right), Y\right\rangle=\left\langle\Xi\left(X_{1}\right),\left[Y_{1}, b\right]\right\rangle=-\left\langle\Xi\left(Y_{1}\right),\left[b, X_{1}\right]\right\rangle-\left\langle\Xi(b),\left[X_{1}, Y_{1}\right]\right\rangle=\left\langle\Xi\left(Y_{1}\right), X\right\rangle,
$$

which proves that $\Gamma_{b}$ is symmetric.

Theorem 7. The assumptions and notations are the same as those in Proposition 14. For each $b \in \Omega$, there exists on the vector subspace $F_{b}=[\mathcal{G}, b]$ of elements $X \in \mathcal{G}$ which can be written $X=\left[X_{1}, b\right]$ for some $X_{1} \in \mathcal{G}$, a symmetric negative bilinear form $\Gamma_{b}$ given by

$$
\Gamma_{b}(X, Y)=\left\langle\Theta_{b}\left(X_{1}\right), Y\right\rangle \text {, with } X_{1} \in \mathcal{G}, X=\left[X_{1}, b\right] \in F_{b}, Y \in F_{b},
$$


where $\Theta_{b}: \mathcal{G} \rightarrow \mathcal{G}^{*}$ is the symplectic 1-cocycle defined in Corollary 4.

Proof. We have seen in Corollary 4 that $b \in \operatorname{ker} \Theta_{b}$. The fact that the equality given in the statement above defines indeed a symmetric bilinear form on $F_{b}$ directly follows from Lemma 1 . We only have to prove that this symmetric bilinear form is negative. Let $X \in F_{b}$ and $X_{1} \in \mathcal{G}$ such that $X=\left[X_{1}, b\right]$. Using Proposition 17 and Corollary 3, we have

$$
\begin{aligned}
\Gamma_{b}(X, X) & =\left\langle\Theta_{b}\left(X_{1}\right),\left[X_{1}, b\right]\right\rangle=\left\langle\Theta\left(X_{1}\right)-\operatorname{ad}_{X_{1}}^{*} E_{J}(b),\left[X_{1}, b\right]\right\rangle=\left\langle D E_{J}(b)\left[X_{1}, b\right],\left[X_{1}, b\right]\right\rangle \\
& \leq 0 .
\end{aligned}
$$

The symmetric bilinear form $\Gamma_{b}$ on $F_{b}$ is therefore negative.

Remark 17. The symmetric negative bilinear forms encountered in Theorem 7 and Corollary 3 seem to be linked with the Fisher metric in information geometry discussed in [31,60,61].

\subsection{Examples of Generalized Gibbs States}

\subsubsection{Action of the Group of Rotations on a Sphere}

The symplectic manifold $(M, \omega)$ considered here is the two-dimensional sphere of radius $R$ centered at the origin $O$ of a three-dimensional oriented Euclidean vector space $\vec{E}$, equipped with its area element as symplectic form. The group $G$ of rotations around the origin (isomorphic to $\mathrm{SO}(3)$ ) acts on the sphere $M$ by a Hamiltonian action. The Lie algebra $\mathcal{G}$ of $G$ can be identified with $\vec{E}$, the fundamental vector field on $M$ associated to an element $\vec{b}$ in $\mathcal{G} \equiv \vec{E}$ being the vector field on $M$ whose value at a point $m \in M$ is given by the vector product $\vec{b} \times \overrightarrow{O m}$. The dual $\mathcal{G}^{*}$ of $\mathcal{G}$ will be too identified with $\vec{E}$, the coupling by duality being given by the Euclidean scalar product. The momentum map $J: M \rightarrow \mathcal{G}^{*} \equiv \vec{E}$ is given by

$$
J(m)=-R \overrightarrow{O m}, \quad m \in M .
$$

Therefore, for any $\vec{b} \in \mathcal{G} \equiv \vec{E}$,

$$
\langle J(m), \vec{b}\rangle=-R \overrightarrow{O m} \cdot \vec{b}
$$

Let $\vec{b}$ be any element in $\mathcal{G} \equiv \vec{E}$. To calculate the partition function $P(\vec{b})$ we choose an orthonormal basis $\left(\overrightarrow{e_{x}}, \overrightarrow{e_{y}}, \overrightarrow{e_{z}}\right)$ of $\vec{E}$ such that $\vec{b}=\|\vec{b}\| \overrightarrow{e_{z}}$, with $\|\vec{b}\| \in \mathbb{R}^{+}$, and we use angular coordinates $(\varphi, \theta)$ on the sphere $M$. The coordinates of a point $m \in M$ are

$$
x=R \cos \theta \cos \varphi, \quad y=R \cos \theta \sin \varphi, \quad z=R \sin \theta .
$$

We have

$$
P(\vec{b})=\int_{0}^{2 \pi}\left(\int_{-\pi / 2}^{\pi / 2} R^{2} \exp (R\|\vec{b}\| \sin \theta \mathrm{d} \theta) \mathrm{d} \varphi=\frac{4 \pi R}{\|\vec{b}\|} \operatorname{sh}(R\|\vec{b}\|) .\right.
$$

The probability density (with respect to the natural area measure on the sphere $M$ ) of the generalized Gibbs state associated to $\vec{b}$ is

$$
\rho_{b}(m)=\frac{1}{P(\vec{b})} \exp (\overrightarrow{O m} \cdot \vec{b}), \quad m \in M .
$$


We observe that $\rho_{b}$ reaches its maximal value at the point $m \in M$ such that $\overrightarrow{O m}=\frac{R \vec{b}}{\|\vec{b}\|}$ and its minimal value at the diametrally opposed point.

\subsubsection{The Galilean Group, Its Lie Algebra and Its Actions}

In view of the presentation, made below, of some physically meaningful generalized Gibbs states for Hamiltonian actions of subgroups of the Galilean group, we recall in this section some notions about the space-time of classical (non-relativistic) mechanics, the Galilean group, its Lie algebra and its Hamiltonian actions. The interested reader will find a much more detailed treatment on these subjects in the book by Souriau [14] or in the recent book by de Saxcé and Vallée [45]. The paper [62] presents a nice application of Galilean invariance in thermodynamics.

The space-time of classical mechanics is a four-dimensional real affine space which, once an inertial reference frame, units of length and time, orthonormal bases of space and time are chosen, can be identified with $\mathbb{R}^{4} \equiv \mathbb{R}^{3} \times \mathbb{R}$ (coordinates $x, y, z, t$ ). The first three coordinates $x, y$ and $z$ can be considered as the three components of a vector $\vec{r} \in \mathbb{R}^{3}$, therefore an element of space-time can be denoted by $(\vec{r}, t)$. However, as the action of the Galilean group will show, the splitting of space-time into space and time is not uniquely determined, it depends on the choice of an inertial reference frame. In classical mechanics, there exists an absolute time, but no absolute space. There exists instead a space (which is an Euclidean affine three-dimensional space) for each value of the time. The spaces for two distinct values of the time should be considered as disjoint.

The space-time being identified with $\mathbb{R}^{3} \times \mathbb{R}$ as explained above, the Galilean group $G$ can be identified with the set of matrices of the form

$$
\left(\begin{array}{ccc}
A & \vec{b} & \vec{d} \\
0 & 1 & e \\
0 & 0 & 1
\end{array}\right) \text {, with } A \in \mathrm{SO}(3), \vec{b} \text { and } \vec{d} \in \mathbb{R}^{3}, e \in \mathbb{R},
$$

the vector space $\mathbb{R}^{3}$ being oriented and endowed with its usual Euclidean structure, the matrix $A \in \mathrm{SO}(3)$ acting on it.

The action of the Galilean group $G$ on space-time, identified as indicated above with $\mathbb{R}^{3} \times \mathbb{R}$, is the affine action

$$
\left(\begin{array}{c}
\vec{r} \\
t \\
1
\end{array}\right) \mapsto\left(\begin{array}{ccc}
A & \vec{b} & \vec{d} \\
0 & 1 & e \\
0 & 0 & 1
\end{array}\right)\left(\begin{array}{c}
\vec{r} \\
t \\
1
\end{array}\right)=\left(\begin{array}{c}
A \vec{r}+t \vec{b}+\vec{d} \\
t+e \\
1
\end{array}\right)
$$

The Lie algebra $\mathcal{G}$ of the Galilean group $G$ can be identified with the space of matrices of the form

$$
\left(\begin{array}{ccc}
j(\vec{\omega}) & \vec{\beta} & \vec{\delta} \\
0 & 0 & \varepsilon \\
0 & 0 & 0
\end{array}\right) \text {, with } \vec{\omega}, \vec{\beta} \text { and } \vec{\delta} \in \mathbb{R}^{3}, \varepsilon \in \mathbb{R}
$$

We have denoted by $j(\vec{\omega})$ the $3 \times 3$ skew-symmetric matrix

$$
j(\vec{\omega})=\left(\begin{array}{ccc}
0 & -\omega_{z} & \omega_{y} \\
\omega_{z} & 0 & -\omega_{x} \\
-\omega_{y} & \omega_{x} & 0
\end{array}\right) .
$$

The matrix $j(\vec{\omega})$ is an element in the Lie algebra so(3), and its action on a vector $\vec{r} \in \mathbb{R}^{3}$ is given by the vector product

$$
j(\vec{\omega}) \vec{r}=\vec{\omega} \times \vec{r} .
$$


Let us consider a mechanical system made by a point particle of mass $m$ whose position and velocity at time $t$, in the reference frame allowing the identification of space-time with $\mathbb{R}^{3} \times \mathbb{R}$, are the vectors $\vec{r}$ and $\vec{v} \in \mathbb{R}^{3}$. The action of an element of the Galilean group on $\vec{r}, \vec{v}$ and $t$ can be written as

$$
\left(\begin{array}{cc}
\vec{r} & \vec{v} \\
t & 1 \\
1 & 0
\end{array}\right) \mapsto\left(\begin{array}{ccc}
A & \vec{b} & \vec{d} \\
0 & 1 & e \\
0 & 0 & 1
\end{array}\right)\left(\begin{array}{cc}
\vec{r} & \vec{v} \\
t & 1 \\
1 & 0
\end{array}\right)=\left(\begin{array}{cc}
A \vec{r}+t \vec{b}+\vec{d} & A \vec{v}+\vec{b} \\
t+e & 1 \\
1 & 0
\end{array}\right)
$$

Souriau has shown in his book [14] that this action is Hamiltonian, with the map J, defined on the evolution space of the particle, with value in the dual $\mathcal{G}^{*}$ of the Lie algebra $\mathcal{G}$ of the Galilean group, as momentum map the formula

$$
J(\vec{r}, t, \vec{v}, m)=m\left(\vec{r} \times \vec{v}, \vec{r}-t \vec{v}, \vec{v}, \frac{1}{2}\|\vec{v}\|^{2}\right) .
$$

Let $b=\left(\begin{array}{ccc}j(\vec{\omega}) & \vec{\beta} & \vec{\delta} \\ 0 & 0 & \varepsilon \\ 0 & 0 & 0\end{array}\right)$ be an element in $\mathcal{G}$. Its coupling with $J(\vec{r}, t, \vec{v}, m) \in \mathcal{G}^{*}$ is given by

$$
\langle J(\vec{r}, t, \vec{v}, m), b\rangle=m\left(\vec{\omega} \cdot(\vec{r} \times \vec{v})-(\vec{r}-t \vec{v}) \cdot \vec{\beta}+\vec{v} \cdot \vec{\delta}-\frac{1}{2}\|\vec{v}\|^{2} \varepsilon\right) .
$$

\subsubsection{One-Parameter Subgroups of the Galilean Group}

In his book [14], Souriau has shown that when the considered Lie group action is the action of the full Galilean group on the space of motions of an isolated mechanical system, the open subset $\Omega$ of the Lie algebra $\mathcal{G}$ of the Galilean group on which the conditions specified in Section 7.2 are satisfied is empty. In other words, generalized Gibbs states of the full Galilean group do not exist. However, generalized Gibbs states for one-parameter subgroups of the Galilean group do exist which have an interesting physical meaning.

Let us consider an element $b$ of $\mathcal{G}$ such that in its matrix expression (expression (9) above) we have $\varepsilon \neq 0$. The one-parameter subgroup $G_{1}$ of the Galilean group generated by $b$ is the set of matrices $\exp (\tau b)$, with $\tau \in \mathbb{R}$. We have

$$
\exp (\tau b)=\left(\begin{array}{ccc}
A(\tau) & \vec{b}(\tau) & \vec{d}(\tau) \\
0 & 1 & \tau \varepsilon \\
0 & 0 & 1
\end{array}\right)
$$

with

$$
\begin{aligned}
& A(\tau)=\exp (\tau j(\vec{\omega})), \\
& \vec{b}(\tau)=\left(\sum_{n=1}^{\infty} \frac{\tau^{n}}{n !}(j(\vec{\omega}))^{n-1}\right) \vec{\beta}, \\
& \vec{d}(\tau)=\left(\sum_{n=1}^{\infty} \frac{\tau^{n}}{n !}(j(\vec{\omega}))^{n-1}\right) \vec{\delta}+\varepsilon\left(\sum_{n=2}^{\infty} \frac{\tau^{n}}{n !}(j(\vec{\omega}))^{n-2}\right) \vec{\beta},
\end{aligned}
$$

with the usual convention that $(j(\vec{\omega}))^{0}$ is the unit matrix.

The physical meaning of this one-parameter subgroup of the Galilean group can be understood as follows. Let us call fixed the affine Euclidean reference frame of space $\left(O, \overrightarrow{e_{x}}, \overrightarrow{e_{y}}, \overrightarrow{e_{z}}\right)$ used to represent, at time $t=0$, a point in space by a vector $\vec{r}$ or by its three components $x, y$ and $z$. Let us set $\tau=\frac{t}{\varepsilon}$. 
For each time $t \in \mathbb{R}$, the action of $A(\tau)=A\left(\begin{array}{c}t \\ \varepsilon\end{array}\right)$ maps the fixed reference frame $\left(O, \overrightarrow{e_{x}}, \overrightarrow{e_{y}}, \overrightarrow{e_{z}}\right)$ onto another affine Euclidean reference frame $\left(O(t), \overrightarrow{e_{x}}(t), \overrightarrow{e_{y}}(t), \overrightarrow{e_{z}}(t)\right)$, which we call the moving reference frame. The velocity and the acceleration of the relative motion of the moving reference frame with respect to the fixed reference frame is given, at time $t=0$, by the fundamental vector field associated to the element $b$ of the Lie algebra $\mathcal{G}$ of the Galilean group: we see that each point in space has a motion composed of a rotation around the axis through $O$ parallel to $\vec{w}$, at an angular velocity $\frac{\|\vec{\omega}\|}{\varepsilon}$, and simultaneously a uniformly accelerated motion of translation at an initial velocity $\frac{\vec{\delta}}{\varepsilon}$ and acceleration $\frac{\vec{\beta}}{\varepsilon}$. At time $t$, the velocity and acceleration of the moving reference frame with respect to its instantaneous position at that time can be described in a similar manner, but instead of $O, \vec{\omega}, \vec{\beta}$ and $\vec{\delta}$ we must use the corresponding transformed elements by the action of $A(\tau)=A\left(\frac{t}{\varepsilon}\right)$.

\subsubsection{A Gas Contained in a Moving Vessel}

We consider a mechanical system made by a gas of $N$ point particles, indexed by $i \in\{1,2, \ldots, N\}$, contained in a vessel with rigid, undeformable walls, whose motion in space is given by the action of the one-parameter subgroup $G_{1}$ of the Galilean group made by the $A\left(\frac{t}{\varepsilon}\right)$, with $t \in \mathbb{R}$, above described. We denote by $m_{i}, \overrightarrow{r_{i}}(t)$ and $\overrightarrow{v_{i}}(t)$ the mass, position vector and velocity vector, respectively, of the $i$-th particle at time $t$. Since the motion of the vessel containing the gas is precisely given by the action of $G_{1}$, the boundary conditions imposed to the system are invariant by that action, which leaves invariant the evolution space of the mechanical system, is Hamiltonian and projects onto a Hamiltonian action of $G_{1}$ on the symplectic manifold of motions of the system. We can therefore consider the generalized Gibbs states of the system, as discussed in Section 7.1. We must evaluate the momentum map $J$ of that action and its coupling with the element $b \in \mathcal{G}$. As in Section 6.3.1 we will neglect, for that evaluation, the contributions of the collisions of the particles between themselves and with the walls of the vessel. The momentum map can therefore be evaluated as if all particles were free, and its coupling $\langle J, b\rangle$ with $b$ is the sum $\sum_{i=1}^{N}\left\langle J_{i}, b\right\rangle$ of the momentum map $J_{i}$ of the $i$-th particle, considered as free, with $b$. We have

$$
\left\langle J_{i}\left(\overrightarrow{r_{i}}, t, \overrightarrow{v_{i}}, m_{i}\right), b\right\rangle=m_{i}\left(\vec{\omega} \cdot\left(\overrightarrow{r_{i}} \times \overrightarrow{v_{i}}\right)-\left(\overrightarrow{r_{i}}-t \overrightarrow{v_{i}}\right) \cdot \vec{\beta}+\overrightarrow{v_{i}} \cdot \vec{\delta}-\frac{1}{2}\left\|\overrightarrow{v_{i}}\right\|^{2} \varepsilon\right) .
$$

Following Souriau [14], Chapter IV, pp. 299-303, we observe that $\left\langle J_{i}, b\right\rangle$ is invariant by the action of $G_{1}$. We can therefore define $\overrightarrow{r_{i 0}}, t_{0}$ and $\overrightarrow{v_{i 0}}$ by setting

$$
\left(\begin{array}{cc}
\overrightarrow{r_{i 0}} & \overrightarrow{v_{i 0}} \\
t_{0} & 1 \\
1 & 0
\end{array}\right)=\exp \left(-\frac{t}{\varepsilon} b\right)\left(\begin{array}{cc}
\overrightarrow{r_{i}} & \overrightarrow{v_{i}} \\
t & 1 \\
1 & 0
\end{array}\right)
$$

and write

$$
\left\langle J_{i}\left(\overrightarrow{r_{i}}, t, \overrightarrow{v_{i}}, m_{i}\right), b\right\rangle=\left\langle J_{i}\left(\overrightarrow{r_{i 0}}, t_{0}, \overrightarrow{v_{i 0}}, m_{i}\right), b\right\rangle .
$$

The vectors $\overrightarrow{r_{i 0}}$ and $\overrightarrow{v_{i 0}}$ have a clear physical meaning: they are the vectors $\overrightarrow{r_{i}}$ and $\overrightarrow{v_{i}}$ as seen by an observer moving with the moving affine Euclidean reference frame $\left(O(t), \overrightarrow{e_{x}}(t), \overrightarrow{e_{y}}(t), \overrightarrow{e_{z}}(t)\right)$. Moreover, as can be easily verified, $t_{0}=0$ of course. We therefore have

$$
\begin{aligned}
\left\langle J_{i}\left(\overrightarrow{r_{i}}, t, \overrightarrow{v_{i}}, m_{i}\right), b\right\rangle & =m_{i}\left(\vec{\omega} \cdot\left(\overrightarrow{r_{i 0}} \times \overrightarrow{v_{i 0}}\right)-\overrightarrow{r_{i 0}} \cdot \vec{\beta}+\overrightarrow{v_{i 0}} \cdot \vec{\delta}-\frac{1}{2}\left\|\overrightarrow{v_{i 0}}\right\|^{2} \varepsilon\right) \\
& =m_{i}\left(\overrightarrow{v_{i 0}} \cdot\left(\vec{\omega} \times \overrightarrow{r_{i 0}}+\vec{\delta}\right)-\overrightarrow{r_{i 0}} \cdot \vec{\beta}-\frac{1}{2}\left\|\overrightarrow{v_{i 0}}\right\|^{2} \varepsilon\right)
\end{aligned}
$$


where we have used the well known property of the mixed product

$$
\vec{\omega} \cdot\left(\overrightarrow{r_{i 0}} \times \overrightarrow{v_{i 0}}\right)=\overrightarrow{v_{i 0}} \cdot\left(\vec{\omega} \times \overrightarrow{r_{i 0}}\right)
$$

Let us set

$$
\vec{U}^{*}=\frac{1}{\varepsilon}\left(\vec{\omega} \times \overrightarrow{r_{i 0}}+\vec{\delta}\right)
$$

Using $\overrightarrow{v_{i 0}}-\vec{U}^{*}$ and $\vec{U}^{*}$ instead of $\overrightarrow{v_{i 0}}$, we can write

$$
\left\langle J_{i}\left(\overrightarrow{r_{i}}, t, \overrightarrow{v_{i}}, m_{i}\right), b\right\rangle=m_{i} \varepsilon\left(-\frac{1}{2}\left\|\overrightarrow{v_{i 0}}-\vec{U}^{*}\right\|^{2}-\overrightarrow{r_{i 0}} \cdot \frac{\vec{\beta}}{\varepsilon}+\frac{1}{2}\left\|\vec{U}^{*}\right\|^{2}\right) .
$$

We observe that the vector $\vec{U}^{*}$ only depends on $\varepsilon, \vec{\omega}, \vec{\delta}$, which are constants once the element $b \in \mathcal{G}$ is chosen, and of $\overrightarrow{r_{i 0}}$, not on $\overrightarrow{v_{i 0}}$. It has a clear physical meaning: it is the value of the velocity of the moving affine reference frame with respect to the fixed affine reference frame, at point $\overrightarrow{r_{i 0}}$ seen by an observer linked to the moving reference frame. Therefore the vector $\overrightarrow{w_{i 0}}=\overrightarrow{v_{i 0}}-\vec{U} *$ is the relative velocity of the $i$-th particle with respect to the moving affine reference frame, seen by an observer linked to the moving reference frame.

The three components of $\overrightarrow{r_{i 0}}$ and the three components of $\overrightarrow{p_{i 0}}=m_{i} \overrightarrow{w_{i 0}}$ make a system of Darboux coordinates on the six-dimensional symplectic manilold $\left(M_{i}, \omega_{i}\right)$ of motions of the $i$-th particle. With a slight abuse of notations, we can consider the momentum map $J_{i}$ as defined on the space of motions of the $i$-th particle, instead of being defined on the evolution space of this particle, and write

$$
\left\langle J_{i}\left(\overrightarrow{r_{i 0}}, \overrightarrow{p_{i, 0}}\right), b\right\rangle=-\varepsilon\left(\frac{1}{2 m_{i}}\left\|\overrightarrow{p_{i 0}}\right\|^{2}+m_{i} f_{i}\left(\overrightarrow{r_{i 0}}\right)\right), \overrightarrow{p_{i 0}}=m_{i} \overrightarrow{w_{i 0}}=m_{i}\left(\overrightarrow{v_{i 0}}-\vec{U}^{*}\right),
$$

and

$$
f_{i}\left(\overrightarrow{r_{i 0}}\right)=\overrightarrow{r_{i 0}} \cdot \frac{\vec{\beta}}{\varepsilon}-\frac{1}{2 \varepsilon^{2}}\left\|\vec{\omega} \times \overrightarrow{r_{i 0}}\right\|^{2}-\frac{\vec{\delta}}{\varepsilon} \cdot\left(\frac{\vec{\omega}}{\varepsilon} \times \overrightarrow{r_{i 0}}\right)-\frac{1}{2 \varepsilon^{2}}\|\vec{\delta}\|^{2} .
$$

Equation (10) is well suited for the determination of generalized Gibbs states of the system. Let us set

$$
P_{i}(b)=\int_{M_{i}} \exp \left(-\left\langle J_{i}, b\right\rangle\right) \mathrm{d} \lambda_{\omega_{i}}, \quad E_{J_{i}}(b)=\frac{1}{P_{i}(b)} \int_{M_{i}} J_{i} \exp \left(-\left\langle J_{i}, b\right\rangle\right) \mathrm{d} \lambda_{\omega_{i}} .
$$

The integrals in the right hand sides of these equalities converge if and only if $\varepsilon<0$. It means that the matrix $b$ belongs to the subset $\Omega$ of the one-dimensional Lie algebra of the considered one-parameter subgroup $G_{1}$ of the Galilean group on which generalized Gibbs states can be defined if and only if $\varepsilon<0$. Assuming that condition satisfied, we can use Definitions 19. The generalized Gibbs state determined by $b$ has the smooth density, with respect to the Liouville measure $\prod_{i=1}^{N} \lambda_{\omega_{i}}$ on the symplectic manifold of motions $\Pi_{i=1}^{N}\left(M_{i}, \omega_{i}\right)$,

$$
\rho(b)=\prod_{i=1}^{N} \rho_{i}(b), \quad \text { with } \rho_{i}(b)=\frac{1}{P_{i}(b)} \exp \left(-\left\langle J_{i}, b\right\rangle\right) .
$$

The partition function, whose expression is

$$
P(b)=\prod_{i=1}^{N} P_{i}(b),
$$

can be used, with the help of the formulae given in Section 7.2, to determine all the generalized thermodynamic functions of the gas in a generalized thermodynamic equilibrium state. 


\section{Remark 18.}

1. The physical meaning of the parameter $\varepsilon$ which appears in the expression of the matrix $b$ is clearly apparent in expression (10) of $\left\langle J_{i}, b\right\rangle$ :

$$
\varepsilon=-\frac{1}{k T},
$$

$T$ being the absolute temperature and $k$ the Boltzmann's constant.

2. The same expression (10) shows that the relative motion of the gas with respect to the moving vessel in which it is contained, seen by an observer linked to that moving vessel, is described by a Hamiltonian system in which the kinetic and potential energies of the $i$-th particle are, respectively, $\frac{1}{2 m_{i}}\left\|\overrightarrow{p_{i 0}}\right\|^{2}$ and $m_{i} f_{i}\left(\overrightarrow{r_{i 0}}\right)$. This result can be obtained in another way: by deriving the Hamiltonian which governs the relative motion of a mechanical system with respect to a moving frame, as used by Jacobi [63] to determine the famous Jacobi integral of the restricted circular three-body problem (in which two big planets move on concentric circular orbits around their common center of mass, and a third planet of negligible mass moves in the gravitational field created by the two big planets).

3. The generalized Gibbs state of the system imposes to the various parts of the system, i.e., to the various particles, to be at the same temperature $T=-\frac{1}{k \varepsilon}$ and to be statistically at rest in the same moving reference frame.

\subsubsection{Three Examples}

1. Let us set $\vec{\omega}=0$ and $\vec{\beta}=0$. The motion of the moving vessel containing the gas (with respect to the so called fixed reference frame) is a translation at a constant velocity $\frac{\vec{\delta}}{\varepsilon}$. The function $f_{i}\left(\overrightarrow{r_{i 0}}\right)$ is then a constant. In the moving reference frame, which is an inertial frame, we recover the thermodynamic equilibrium state of a monoatomic gas discussed in Section 6.3.1.

2. Let us set now $\vec{\omega}=0$ and $\vec{\delta}=0$. The motion of the moving vessel containing the gas (with respect to the so called fixed reference frame) is now an uniformly accelerated translation, with acceleration $\frac{\vec{\beta}}{\varepsilon}$. The function $f_{i}\left(\overrightarrow{r_{i 0}}\right)$ now is

$$
f_{i}\left(\overrightarrow{r_{i 0}}\right)=\overrightarrow{r_{i 0}} \cdot \frac{\vec{\beta}}{\varepsilon}
$$

In the moving reference frame, which is no more inertial, we recover the thermodynamic equilibrium state of a monoatomic gas in a gravity field $\vec{g}=-\frac{\vec{\beta}}{\varepsilon}$ discussed in Section 6.3.2.

3. Let us now set $\vec{\omega}=\omega \overrightarrow{e_{z}}, \vec{\beta}=0$ and $\vec{\delta}=0$. The motion of the moving vessel containing the gas (with respect to the so called fixed reference frame) is now a rotation around the coordinate $z$ axis at a constant angular velocity $\frac{\omega}{\varepsilon}$. The function $f_{i}\left(\overrightarrow{r_{i 0}}\right)$ is now

$$
f_{i}\left(\overrightarrow{r_{i 0}}\right)=-\frac{\omega^{2}}{2 \varepsilon^{2}}\left\|\overrightarrow{e_{z}} \times \overrightarrow{r_{i 0}}\right\|^{2}
$$

The length $\Delta=\left\|\overrightarrow{e_{z}} \times \overrightarrow{r_{i, 0}}\right\|$ is the distance between the $i$-th particle and the axis of rotation of the moving frame (the coordinate $z$ axis). Moreover, we have seen that $\varepsilon=\frac{-1}{k T}$. Therefore in the generalized Gibbs state, the probability density $\rho_{i}(b)$ of presence of the $i$-th particle in its symplectic manifold of motion $M_{i}, \omega_{i}$, with respect to the Liouville measure $\lambda_{\omega_{i}}$, is

$$
\rho_{i}(b)=\frac{1}{P_{i}(b)} \exp \left(-\left\langle J_{i}, b\right\rangle\right)=\text { Constant } \cdot \exp \left(-\frac{1}{2 m_{i} k T}\left\|\overrightarrow{p_{i 0}}\right\|^{2}+\frac{m_{i}}{2 k T}\left(\frac{\omega}{\varepsilon}\right)^{2} \Delta^{2}\right) .
$$


This formula describes the behaviour of a gas made of point particles of various masses in a centrifuge rotating at a constant angular velocity $\frac{\omega}{\varepsilon}$ : the heavier particles concentrate farther from the rotation axis than the lighter ones.

\subsubsection{Other Applications of Generalized Gibbs States}

Applications of generalized Gibbs states in thermodynamics of continua, with the use of affine tensors, are presented in the papers by de Saxcé [64,65].

Several applications of generalized Gibbs states of subgroups of the Poincaré group were considered by Souriau. For example, he presents in his book [14], Chapter IV, p. 308, a generalized Gibbs which describes the behaviour of a gas in a relativistic centrifuge, and in his papers $[15,16]$, very nice applications of such generalized Gibbs states in Cosmology.

Acknowledgments: I address my thanks to Alain Chenciner for his interest and his help to study the works of Claude Shannon, to Roger Balian for his comments and his explanations about thermodynamic potentials, and to Frédéric Barbaresco for his kind invitation to participate in the GSI 2015 conference and his encouragements. My warmest thanks to the anonymous referees whose very careful and benevolent reading of my work allowed me to correct several mistakes and to improve this paper.

Conflicts of Interest: The author declares no conflict of interest.

\section{References}

1. Abraham, R.; Marsden, J.E. Foundations of Mechanics, 2nd ed.; American Chemical Society: Washington, DC, USA, 1978.

2. Arnold, V.I. Mathematical Methods of Classical Mechanics, 2nd ed.; Springer: Berlin/Heidelberg, Germany, 1978.

3. Cannas da Silva, A. Lectures on Symplectic Geometry; Springer: Berlin/Heidelberg, Germany, 2001.

4. Guillemin, V.; Sternberg, S. Symplectic Techniques in Physics; Cambridge University Press: Cambridge, UK, 1984.

5. Holm, D. Geometric Mechanics, Part I: Dynamics ans Symmetry; World Scientific: Singapore, 2008.

6. Holm, D. Geometric Mechanics, Part II: Rotating, Translating and Rolling; World Scientific: Singapore, 2008.

7. Iglesias, P. Symétries et Moment; Éditions Hermann: Paris, France, 2000. (In French)

8. Laurent-Gengoux, C.; Pichereau, A.; Vanhaecke, P. Poisson Structures; Springer: Berlin/Heidelberg, Germany, 2013.

9. Libermann, P.; Marle, C.-M. Symplectic Geometry and Analytical Mechanics; Springer: Berlin/Heidelberg, Germany, 1987.

10. Ortega, J.-P.; Ratiu, T.-S. Momentum Maps and Hamiltonian Reduction; Birkhäuser: Boston, MA, USA; Basel, Switzerland; Berlin, Germany, 2004.

11. Vaisman, I. Lectures on the Geometry of Poisson Manifolds; Springer: Berlin/Heidelberg, Germany, 1994.

12. Marle, C.-M. Symmetries of hamiltonian systems on symplectic and poisson manifolds. In Similarity and Symmetry Methods, Applications in Elasticity and Mechanics of Materials; Ganghoffer, J.-F., Mladenov, I., Eds.; Springer: Berlin/Heidelberg, Germany, 2014; pp. 183-269.

13. Souriau, J.-M. Définition covariante des équilibres thermodynamiques. Supplemento al Nuovo Cimento 1966, 4 , 203-216. (In French)

14. Souriau, J.-M. Structure des Systèmes Dynamiques; Dunod: Malakoff, France, 1969. (In French)

15. Souriau, J.-M. Mécanique Statistique, Groupes de Lie et Cosmologie. In Géométrie Symplectique et Physique Mathématique; CNRS Éditions: Paris, France, 1974; pp. 59-113. (In French)

16. Souriau, J.-M. Géométrie symplectique et Physique mathématique. In Deux Conférences de Jean-Marie Souriau, Colloquium de la Société Mathématique de France, Paris, France, 19 February-12 November 1975. (In French)

17. Souriau, J.-M. Mécanique Classique et Géométrie Symplectique; Dunod: Malakoff, France, 1984. (In French)

18. Mackey, G.W. The Mathematical Foundations of Quantum Mechanics; W. A. Benjamin, Inc.: New York, NY, USA, 1963. 
19. Newton, I. Philosophia Naturalis Principia Mathematica; Translated in French by Émilie du Chastelet (1756); London, UK, 1687. (In French)

20. Lagrange, J.L. Mécanique Analytique, 1st ed.; La veuve de Saint-Pierre: Paris, France, 1808; reprinted by Jacques Gabay: Paris, France, 1989. (In French)

21. Hamilton, W.R. On a general method in Dynamics. In Sir William Rowan Hamilton Mathematical Works, Volume II; Cambridge University Press: Cambridge, UK, 1940; pp. 247-308.

22. Hamilton, W.R. Second essay on a general method in Dynamics. In Sir William Rowan Hamilton Mathematical Works, Volume II; Cambridge University Press: Cambridge, UK, 1940; pp. 95-144.

23. Bérest, P. Calcul des Variations Application à la Mécanique et à la Physique; Ellipses/Éditions Marketing: Paris, France, 1997. (In French)

24. Bourguignon, J.-P. Calcul Variationnel; Éditions de l'École Polytechnique: Paris, France, 1991. (In French)

25. Lanczos, C.S. The Variational Principles of Mechanics, 4th ed.; Reprinted by Dover, New York, 1970; University of Toronto Press: Toronto, ON, Canada, 1970.

26. Malliavin, P. Géométrie Différentielle Intrinsèque; Éditions Hermann: Paris, France, 1972. (In French)

27. Sternberg, S. Lectures on Differential Geometry; Prentice-Hall: Upper Saddle River, NJ, USA, 1964.

28. Kosmann-Schwarzbach, Y. The Noether Theorems; Springer: Berlin/Heidelberg, Germany, 2011.

29. Poincaré, H. Sur une forme nouvelle des équations de la Méanique. C. R. Acad. Sci. 1901, 7, 369-371.

30. Marle, C.-M. On Henri Poincaré's note "Sur une forme nouvelle des équations de la Mécanique". J. Geom. Symmetry Phys. 2013, 29, 1-38.

31. Barbaresco, F. Symplectic structure of information geometry: Fisher metric and euler-poincaré equation of souriau lie group thermodynamics. In Geometric Science of Information: Second International Conference, GSI 2015, Proceedings; Nielsen, F., Barbaresco, F., Eds.; Lecture Notes in Computer Science; Springer: Berlin/Heidelberg, Germany, 2015; Volume 9389, pp. 529-540. (In French)

32. Lagrange, J.-L. Mémoire sur la Théorie Générale de la Variation des Constantes Arbitraires Dans Tous les Problèmes de Mécanique; Lu le 13 mars 1809 à l'Institut de France; Dans CEuvres de Lagrange; Gauthier-Villars: Paris, France, 1877; Volume VI, pp. 771-805. (In French)

33. Lagrange, J.-L. Second Mémoire sur la Théorie de la Variation des Constantes Arbitraires Dans les Problèmes de Mécanique; Gauthier-Villars: Paris, France, 1877; Volume VI, pp. 809-816. (In French)

34. Tulczyjew, W.M. Hamiltonian systems, Lagrangian systems and the Legendre transformation. Symp. Math. 1974, 14, 247-258.

35. Tulczyjew, W.M. Geometric Formulations of Physical Theories; Monographs and Textbooks in Physical Science; Bibliopolis: Napoli, Italy, 1989.

36. Lichnerowicz, A. Les variétés de Poisson et leurs algèbres de Lie associées. J. Differ. Geom. 1977, 12, $253-300$. (In French)

37. Lichnerowicz, A. Les variétés de Jacobi et leurs algèbres de Lie associées. J. Math. Pures Appl. 1979, 57, 453-488. (In French)

38. Kirillov, A. Local lie algebras. Russ. Math. Surv. 1976, 31, 55-75.

39. Poisson, S.D. Sur la variation des constantes arbitraires dans les questions de mécanique. Mémoire lu le 16 octobre 1809 à l'Institut de France. Journal de L'École Polytechnique quinzième cahier, tome VIII, 266-344. (In French)

40. Koszul, J.-L. Crochet de Schouten-Nijenhuis et cohomologie. In É. Cartan et les Mathématiques D'aujourd'hui; Astérisque, numéro hors série, Société Mathématique de France: Paris, France, 1985; pp. 257-271. (In French)

41. Marle, C.-M. Calculus on Lie algebroids, Lie groupoids and Poisson manifolds. Dissertationes Mathematicae 457, Institute of Mathematics, Polish Academy of Sciences (Warszawa). 2008, arXiv:0806.0919.

42. Weinstein, A. The local structure of Poisson manifolds. J. Differ. Geom. 1983, 18, 523-557.

43. Marsden, J.E.; Weinstein, A. Reduction of symplectic manifolds with symmetry. Rep. Math. Phys. 1974, 5, 121-130.

44. Meyer, K. Symmetries and integrals in mechanics. In Dynamical Systems; Peixoto, M., Ed.; Academic Press, New York, NY, USA, 1973; pp. 259-273.

45. De Saxcé, G.; Vallée, C. Galilean Mechanics and Thermodynamics of Continua; John Wiley \& Sons: Hoboken, NJ, USA, 2016.

46. Boltzmann, L.E. Leçons sur la Théorie des gaz. Available online: http://iris.univ-lille1.fr/handle/1908/1523 (accessed on 11 October 2016). (In French) 
47. Shannon, C.E. A mathematical theory of communication. Bell Syst. Tech. J. 1948, 27, 379-423, $623-656$.

48. Jaynes, E.T. Information theory and statistical mechanics. Phys. Rev. 1957, 106, 620-630.

49. Jaynes, E.T. Information theory and statistical mechanics II. Phys. Rev. 1957, 108, 171-190.

50. Balian, R. Information in statistical physics. Stud. Hist. Philos. Mod. Phys. Part B 2005, 36, 323-353.

51. Poincaré, H. Sur le problème des trois corps et les équations de la dynamique. Acta Math. 1890, doi:10.1007/BF02392506. (In French)

52. Kinetic Theory. Available online: http://hyperphysics.phy-astr.gsu.edu/hbase/kinetic/kinthe.html (accessed on 11 October 2016).

53. Gastebois, G. Théorie Cinétique des Gaz. Available online: http://gilbert.gastebois.pagesperso-orange.fr/ java/gaz/gazparfait/theorie_gaz.pdf (accessed on 11 October 2016). (In French)

54. Synge, J.L. The Relativistic Gas; North Holland Publishing Company: Amsterdam, The Netherlands, 1957.

55. Massieu, F. Sur les Fonctions caractéristiques des divers fluides. C. R. Acad. Sci. Paris 1869, 69, 858-862. (In French)

56. Massieu, F. Addition au précédent Mémoire sur les Fonctions caractéristiques. C. R. Acad. Sci. Paris 1869, 69, 1057-1061. (In French)

57. Massieu, F. Thermodynamique. Mémoire sur les Fonctions Caractéristiques des Divers Fluides et sur la Théorie des Vapeurs; Académie des Sciences: Paris, France, 1876; pp. 1-92. (In French).

58. Balian, R. François Massieu et les Potentiels Thermodynamiques; Évolution des Disciplines et Histoire des Découvertes; Académie des Sciences: Paris, France, 2015. (In French)

59. Callen, H.B. Thermodynamics and an Introduction to Thermostatics, 2nd ed.; John Wiley and Sons: New York, NY, USA, 1985.

60. Barbaresco, F. Koszul information geometry and Souriau geometric temperature/capacity of lie group thermodynamics. Entropy 2014, 16, 4521-4565.

61. Barbaresco, F. Geometric theory of heat from Souriau lie groups thermodynamics and koszul hessian geometry: Applications in information geometry for exponential families. Entropy 2016, doi:10.20944/preprints201608.0078.v1.

62. De Saxcé, G.; Vallée, C. Bargmann group, momentum tensor and Galilean invariance of Clausius-Duhem inequality. Int. J. Eng. Sci. 2012, 50, 216-232.

63. Jacobi, C.G.J. Sur le mouvement d'un point et sur un cas particulier du problème des trois corps. C. R. Acad. Sci. 1836, 3, 59-61. (In French)

64. De Saxcé, G. Entropy and structure for the thermodynamic systems. In Geometric Science of Information, Second International Conference GSI 2015 Proceedings; Nielsen, F., Barbaresco, F., Eds.; Lecture Notes in Computer Science; Springer: Berlin/Heidelberg, Germany, 2015; Volume 9389, pp. 519-528.

65. De Saxcé, G. Link between lie group statistical mechanics and thermodynamics of continua. Entropy 2016, doi:10.3390/e18070254.

(C) 2016 by the author; licensee MDPI, Basel, Switzerland. This article is an open access article distributed under the terms and conditions of the Creative Commons Attribution (CC-BY) license (http://creativecommons.org/licenses/by/4.0/). 